\title{
The Subband Modulation: A Joint Power and Rate Allocation Framework for Subband Image and Video Transmission
}

\author{
Haitao Zheng and K. J. Ray Liu, Senior Member, IEEE
}

\begin{abstract}
A new approach of reliable image and video transmission over noisy channels is proposed. For subband decomposed image and video, combined source coding and channel modulation design can achieve high compression efficiency and preferable quality. Further performance gain is obtained by multiresolution modulation as well as a bit-remapping scheme that assigns efficient mapping from each source codeword to channel modulation points. We show that the combined source coding and modulation design outperforms conventional approaches, which design source coding and modulation separately. A simple channel distortion approximation is derived by applying a bitremapping scheme, which allows the power allocation to be employed to further enhance the performance. Compared to the joint source and channel coding with a binary phase shift keying modulation system and fixed modulation with the one-to-one intelligent mapping system, the proposed system performs better in a middle-range signal-to-noise ratio and low channel bandwidth. The simulation is carried out on additive white Gaussian noise channels.
\end{abstract}

Index Terms-Combined source coding and channel modulation, image and video transmission, multiresolution modulation, subband decomposition.

\section{INTRODUCTION}

$\mathbf{R}$ ELIABLE image and video transmission over noisy channels has been a great challenge in multimedia communication, especially for the transmission of large volume data over unreliable and bandwidth-limited channels. In video conference applications, the bandwidth assigned to each user is quite limited, and if a scheme can reduce the bandwidth usage of each user while maintaining preferable quality, the original bandwidth can support more users. Therefore, developing reliable transmission under a given bandwidth constraint has become an important research issue. For certain applications that contain several independent data streams of different importance, one solution is the joint design of the source and channel coder, as well as the modem for all data sets. It aims to allocate different bit rates for source and channel units of different data sets to minimize the overall distortion under a fixed bandwidth constraint. Such an approach can be viewed as an extension of the source bit-allocation scheme presented

Manuscript received January 1, 1998; revised December 17, 1998. This work was supported in part by the National Science Foundation under Young Investigator Award MIP9457397. This paper was recommended by Associate Editor J. Brailean.

The authors are with the Electrical Engineering Department and the Institute for Systems Research, University of Maryland, College Park, MD 20742 USA. Publisher Item Identifier S 1051-8215(99)06191-1. in [1]. There are two different extensions, i.e., the combined source coding and channel coding and the combined source coding and channel modulation.

In [2], joint source and channel coding is implemented on three-dimensional (3-D) subband coding in conjunction with conditional arithmetic coding, rate-compatible punctured convolutional (RCPC) [5] codes, and the binary phase shift keying (BPSK) modulation over the binary symmetric channel model. This approach optimally partitions source and channel coded bits to minimize the distortion measured as mean squared error (MSE) between the original and received video. In [3], an adaptive source-channel video coding scheme is proposed that jointly chooses the source coding rate and the level of protection quantified by the channel coding rate. Such an approach works well for very noisy channels, where channel codes are required to combat channel noise. This approach either increases the total bit rate or decreases the source bit rate if the total bit rate remains fixed. Indeed, for wireless communications, the channel bandwidth is the most precious resource.

In general, the second approach combines the source coder and modulator design by mapping the source quantization codewords directly to the constellation points. In [4], combined subband signal decomposition and dynamic bandwidth allocation on 81-pulse amplitude modulation (PAM) transmission is proposed. The quantization codewords of discrete cosine transform blocks are one-to-one directly mapped to the channel constellation. In [6], the authors employ a joint source coding and multiresolution (MR) modulation design that maps data directly to the constellation and assigns the required power to each point. These two approaches have the same assumption that the constellation size is decided by the cardinality of the source quantization codewords. The modulation rate is equal to the sum of codeword length of all the blocks in [4] and the codeword length of the current block in [6]. In the fine source quantization case, such an assumption results in a large size of the constellation. This requires large transmitted power or channel signal-to-noise ratio (SNR) to maintain the required bit error rate (BER).

It comes to our attention that the aforementioned approaches are suited for Gaussian channels exhibiting low and high channel SNR's, respectively. In this paper, we present an approach that handles the image and video transmission over a noisy channel exhibiting moderate channel SNR (8-22 dB) and tightly limited bandwidth $(0.3-1.0 \mathrm{bpp})$. This is one of 
the most important performance regions in practice, and yet few approaches provided good solutions so far. No channel codes are employed due to the low bandwidth constraint. We attempt to minimize the distortion in the mean square sense by first decomposing the source data to subbands with different perceptual importance [7] and constructing the source coder and channel modulator to provide unequal error protection (UEP) for different subbands. Then the MR modulation with bit remapping allows the UEP to be applied to the codeword bit level.

This paper is organized as follows. Section II presents the motivation for combining source coding and modulation design. We derive the optimization function and discuss the solution in Section III. Section IV describes the characteristics and advantages of the MR modulation and bit-remapping scheme, while a specific computation of error statistics is provided. We simplify the optimization problem in Section V. Section VI carries out the simulation on an additive white Gaussian noise (AWGN) channel as well as the comparisons to the approaches in literature. In Section VII, we conclude this paper.

\section{COMBIned Source CODING AND Modulation}

The combined source and channel coding approach is a classical solution to noisy channel transmission and has been studied extensively. Mathematically, it is equivalent to distributing the given bit budget efficiently among a set of given admissible choices of source coding and channel coding to minimize the overall distortion. Some popular techniques include channel matched source coders [e.g., channel matched vector quantization (CM-VQ)] [8] and source coding with error-correcting codes [2], [3], as well as the intelligent source and channel space mapping design [4], [6]. For subband decomposed source data, combined systems show promising advantages. Since subbands are of different perceptual importance which can be classified by variance, UEP for different subbands achieves better performance compared to equal error protection. Combined systems achieve UEP by employing different source coding and channel coding schemes on different subbands. Define $N$ as the number of subbands generated and $R_{i, s}$ and $R_{i, c}$ as the source rate and channel rate of subband $i$, respectively. The objective is to find $\left\{R_{i, s}, R_{i, c}\right\}_{i=1}^{N}$ to minimize the overall distortion under a fixed overall bit-rate constraint $\sum_{i=1}^{N} R_{i}=\sum_{i=1}^{N}\left(R_{i, s} / R_{i, c}\right)=R_{\text {budget }}$. We observe that the following inter- and intratradeoffs exist.

- Intertradeoffs: The subband rates $\left\{R_{i}=\left(R_{i, s} / R_{i, c}\right)\right\}_{i=1}^{N}$ must be allocated efficiently. Subbands of higher importance should have larger $R_{i, s}$ and better channel protection (smaller $R_{i, c}$ ) and therefore larger $R_{i}$. This would result in lower source and channel distortion of subband $i$. On the other hand, less important subbands are assigned with smaller $R_{i, s}$ and less channel protection (larger $R_{i, c}$ ) and therefore smaller $R_{i}$.

- Intratradeoffs: Even if $R_{i}$ is decided, $R_{i, s}$ and $R_{i, c}$ can still be adjusted to achieve a balance between source and channel distortion. For example, increasing $R_{i, s}$ yields smaller source distortion, but to maintain the same $R_{i}$, we have to increase $R_{i, c}$, which may yield larger channel distortion.

Therefore, the system can adjust a subband's distortion in terms of $R_{i}$ and $\left\{R_{i, s}, R_{i, c}\right\}$ for fixed $R_{i}$. To make the adjustment simple and effective, the source coding and channel coding should match the transmission requirements and the channel conditions as well as each other. Particularly, if the transmission bandwidth is limited, both the source coding and the channel coding should exhibit efficient compression capability in addition to information protection capability. For moderate SNR and low bandwidth channel transmission, we design the system in three steps.

\section{A. Select Source Coder}

In general, the source coding removes as much redundancy from the source signal as possible. A different number of quantization levels will yield different bit rates and distortions. The quantized signal can be compressed using run-length, arithmetic, and Huffman codes [2], i.e., variable- or fixedlength codes. For noisy channel transmission, fixed-length codes show more robustness against transmission error compared to variable-length codes, although they achieve less compression. Variable-length codes have to rely on additional synchronization. If an error causes the loss of sequence synchronization, the codes after the error incident will be lost unless the sequence is resynchronized. Furthermore, the distortion of fixed-length codes is much easier to measure than that of variable-length codes. We use vector quantization (VQ) represented in fixed-length codes in our design.

\section{B. Select Channel Coding and Modulation}

Roughly speaking, channel coding is a process of intelligent redundancy insertion that protects the source codeword against channel noise. Employment of channel protection codes is required for very noisy channels, which exhibit more severe channel distortion than source distortion. For channel SNR's higher than about $8 \mathrm{~dB}$, as in video-conference applications, maintaining video quality under low transmission rates becomes a common requirement. Thus, compression becomes a very important factor in both the source and channel coding design.

Applying channel codes to BPSK modulation is not suitable for transmissions at a low rate, since it induces redundancy. Multilevel modulations offer different compression capabilities as well as different error protections. For example, with SNR equal to $10 \mathrm{~dB}$, QAM-16 modulation can compress the source data by a factor of four with an average BER equal to 0.0787 and the QAM-64 modulation by a factor of six with average BER equal to 0.1525 [9]. For moderate channel SNR's, coded BPSK modulation may yield negligible channel distortion compared to others. However, received data still suffer large distortion due to small source rates. If employing multilevel modulations, channel distortion and source distortion will both exist. Compared to coded BPSK, the source rates will increase without changing the overall rate, which leads to a drop in the source distortion as well as a drop in the distortion between two neighboring codewords. This may compensate the increase 


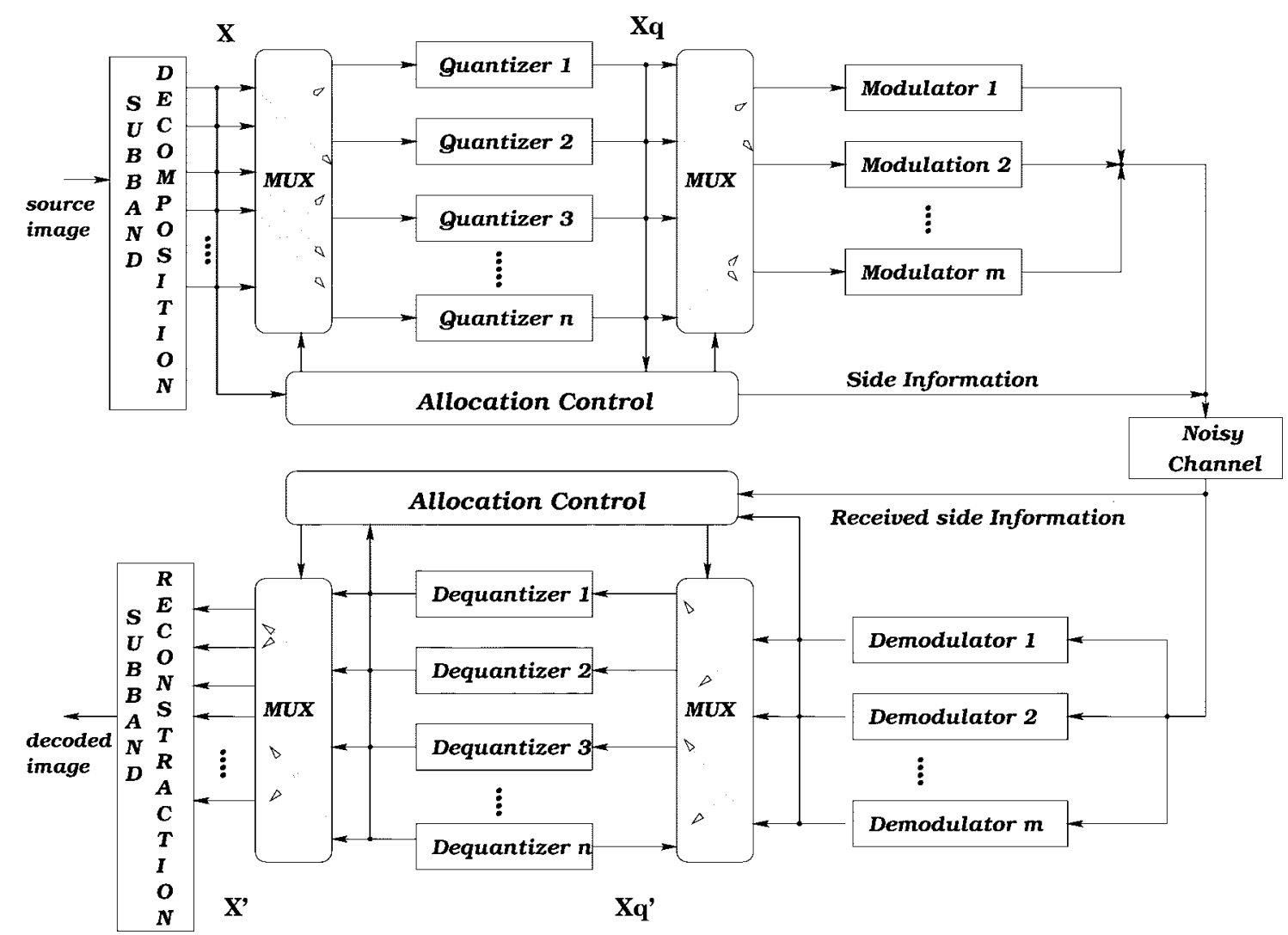

Fig. 1. Block diagram of the combined source coding and modulation based on subband decomposition system.

in error probability. Multilevel modulation also benefits from the flexibility of adjusting the mapping from source code to channel modulation code space according to the source characteristic. Considering the above advantages, we choose to use multilevel modulation over the coded BPSK.

\section{Match Source Coding to Channel Modulation}

After source coding, the input data is turned into a bitstream. The modulator maps these binary digits into a set of corresponding signal waveforms. The assignment of binary bits to waveforms or constellation points is essential to overall performance. It is an interface that connects the source coding and channel modulation together. One preferred mapping scheme is that the binary representations of adjacent constellation points differ by one bit, called Gray encoding. The most likely errors caused by channel noise involve the erroneous selection of an adjacent constellation point to the transmitted constellation point. In such a case, the assignment leads to only a single bit error. Gray encoding only depends on the channel modulation, not source input. It aims to reduce the number of erroneous bits but does not consider where the error happens. If the input bits are of different importance, the mapping and constellation should provide UEP to different bits and have the capability to change the error performance flexibly. Random mapping may cause severe performance degradation as shown in [4].

In this paper, we consider the mapping from source codeword to constellation. For channels exhibiting moderate channel SNR, the transmitted power cannot support constellations of large size, such as PAM-81, QAM-128, etc. Instead, constellations of moderate size such as PAM-4 and QAM-64, are employed. Therefore, source codeword length $R_{i, s}$ is usually larger than modulation rate $R_{i, c}$, e.g., two for PAM-4, four for QAM-16. In such a case, one codeword is mapped to several constellation points instead of one. The one-to-one mapping (one codeword to one constellation point) [6] and multipleto-one (multiple codewords to one constellation point) [4] are not applicable here. We need a one-to-multiple mapping in the proposed system.

The proposed system is shown in Fig. 1. The input image or video is decomposed into several subbands of different importance and vector quantized. The indexes are represented as fixed-length binary codewords and mapped to a set of constellation points at the modulators. The lengths of source codewords and channel modulations are jointly chosen under an overall bit budget. At the receiver, the reverse procedure is performed. Although no channel code is employed, the channel noise effect is reduced by a proper mapping from source code to channel modulation space.

\section{ThE OPTIMIZATION}

In this section, we present the proposed system structure. After deriving the source and channel distortion as the functions of source and channel rates, we formulate the constraint optimization problem and develop a fast solution using Lagrange multiplier.

Without a loss of generality, it is usually assumed that the source quantization error is orthogonal to the channel error and 
that the total distortion is the sum of the source quantization distortion $D_{s}$ and the channel error distortion $D_{c}$ [2], [3]. The source distortion of subband $i, i=1 \ldots N$ is defined as

$$
D_{i, s}\left(R_{i, s}\right)=\sum_{n=0}^{N_{i, s}-1} D\left(x_{n}, Q_{R_{i, s}}^{-1}\left(Q_{R_{i, s}}\left(x_{n}\right)\right)\right)
$$

where $x_{n}$ is the input data number $n, Q_{R_{i, s}}\left(x_{n}\right)$ is the quantized $x_{n}$ at source rate $R_{i, s}$, and $Q_{R_{i, s}}^{-1}\left(x_{q}\right)$ is the dequantized $x_{q}$ at source rate $R_{i, s}$. We also define $N_{i, s}$ as the size of subband $i$, and $D(a, b)$ is MSE between $a$ and $b$. The channel distortion $D_{i, c}$ is determined by both $R_{i, s}$ and $R_{i, c}$

$$
D_{i, c}\left(R_{i, s}, R_{i, c}\right)=\sum_{n=0}^{N_{i, s}-1} \sum_{k=0, k \neq n}^{N_{i, s}-1} P\left(Q_{R_{i, s}}\left(x_{k}\right) \mid Q_{R_{i, s}}\left(x_{n}\right) ;\right.
$$

where $P(a \mid b ; R)$ is the probability of receiving codeword $a$ given the sending of codeword $b$ for modulation of rate $R$.

Due to the subband independence, the total distortion and rate will be the sum of distortion and rate of all the subbands, i.e.,

$$
D_{\text {total }}=\sum_{i=0}^{M-1}\left\{D_{i, s}+D_{i, c}\right\}
$$

and

$$
R_{\mathrm{total}}=\sum_{i=0}^{M-1} R_{i, s} / R_{i, c}
$$

The objective is to find, given the bit budget $R_{\text {budget }}$ to code $N$ independent subbands, the optimal $\left\{R_{i, s}, R_{i, c}\right\}_{i=1}^{N}$ to minimize the overall distortion

$$
\begin{array}{ll}
\text { Min } & D_{\text {total }} \\
\text { subject to } & R_{\text {total }}<R_{\text {budget }} .
\end{array}
$$

The solution to the above constraint minimization problem is similar to the one developed in [11]. Due to the additivity of the rate and distortion measures over independent subbands, the constrained problem can be converted into an equivalent unconstrained problem by merging rate and distortion through the Lagrange multiplier $\lambda$. Thus, the constrained problem turns out to be the minimization of the Lagrangian cost function $J(\lambda)$ given by

$$
\begin{aligned}
J(\lambda) & =D_{\text {total }}+\lambda R_{\text {total }} \\
& =\sum_{i=0}^{M-1}\left(D_{i, s}+D_{i, c}\right)+\lambda\left\{\sum_{i=0}^{M-1} R_{i, s} / R_{i, c}\right\} .
\end{aligned}
$$

It can be shown that for a given $\lambda$, the optimization can be solved for each $M$ independent subband [2], [11]. Therefore, it reduces to solving $\operatorname{Min}_{\left(R_{i, s}, R_{i, c}\right)}\left\{D_{i, s}+D_{i, c}+\lambda R_{i, s} / R_{i, c}\right\}$ for each $i$. Using integer programming, one can obtain the best selection of modulation and quantization for a given $\lambda$. Under the condition that the number of quantization rates and modulation rates take on finite integer values, the exhaustive search can be used. The optimal $\lambda$ is the slope that maximizes the cost function $W(\lambda)$ defined by [11]

$$
W(\lambda)=\operatorname{Min}\left(D_{\text {total }}+\lambda R_{\text {total }}\right)-\lambda R_{\text {budget }}
$$

which is a concave function of $\lambda$. The optimal $\lambda$ can be found using a fast convex searching algorithm.

\section{Multiresolution Modulation ANd Bit Remapping}

Choosing appropriate modulation with efficient power consumption, high compression, and reliable error protection is a crucial step in the proposed system. Among various multilevel modulation schemes, the MR modulation [6] displays nice properties. This section provides a brief description of the MR modulation as well as a specific computation of error performance. We also develop a bit-remapping scheme that maps a single source codeword to multiple constellation points to provide unequal error protection, in contrast to the one-toone mapping in [6].

\section{A. Multiresolution Modulation}

It is well known that for the same transmitted power, modulations with different rates correspond to different minimum distances and therefore have different error performance. Such a property allows us to assign unequal error protection to different subbands by varying their modulation rates. This is a subband-level UEP. In addition, the binary codeword can be arranged in such a way that the most significant bits (MSB's) of a codeword are far more important than the least significant bits (LSB's). We can further improve the performance by applying UEP to bit level.

It was shown in [12] that different error performance exists inside the constellation, where different subchannels in terms of bits at different locations of binary representation of modulation points have different BER curves. This property can be very advantageous because this UEP might remove the need for complex forward error-correction codes [12]. This advantage can be use to provide UEP to the bits of a source codeword. However, UEP only achieves better performance than equal error protection if the error performances are assigned properly according to the source signal characteristic. The conventional modulation constellation [12] has the limitation that the subchannel error performance is fixed. Implementation of UEP by means of modulation design was also presented in [13] and [14], where the constellation consists of uniformly spaced signal points, which embeds the lower rate constellation in a higher one. Inside each constellation point, the bits that represent the lower rate constellation receive higher protection than the bits that represent the higher rate constellation. Similarly, the error performances are decided by the constellation size. In [6], an MR modulation for multiuser communications was developed. It is modeled as "clouds" of "satellites" points. Fig. 2 illustrates the MR 

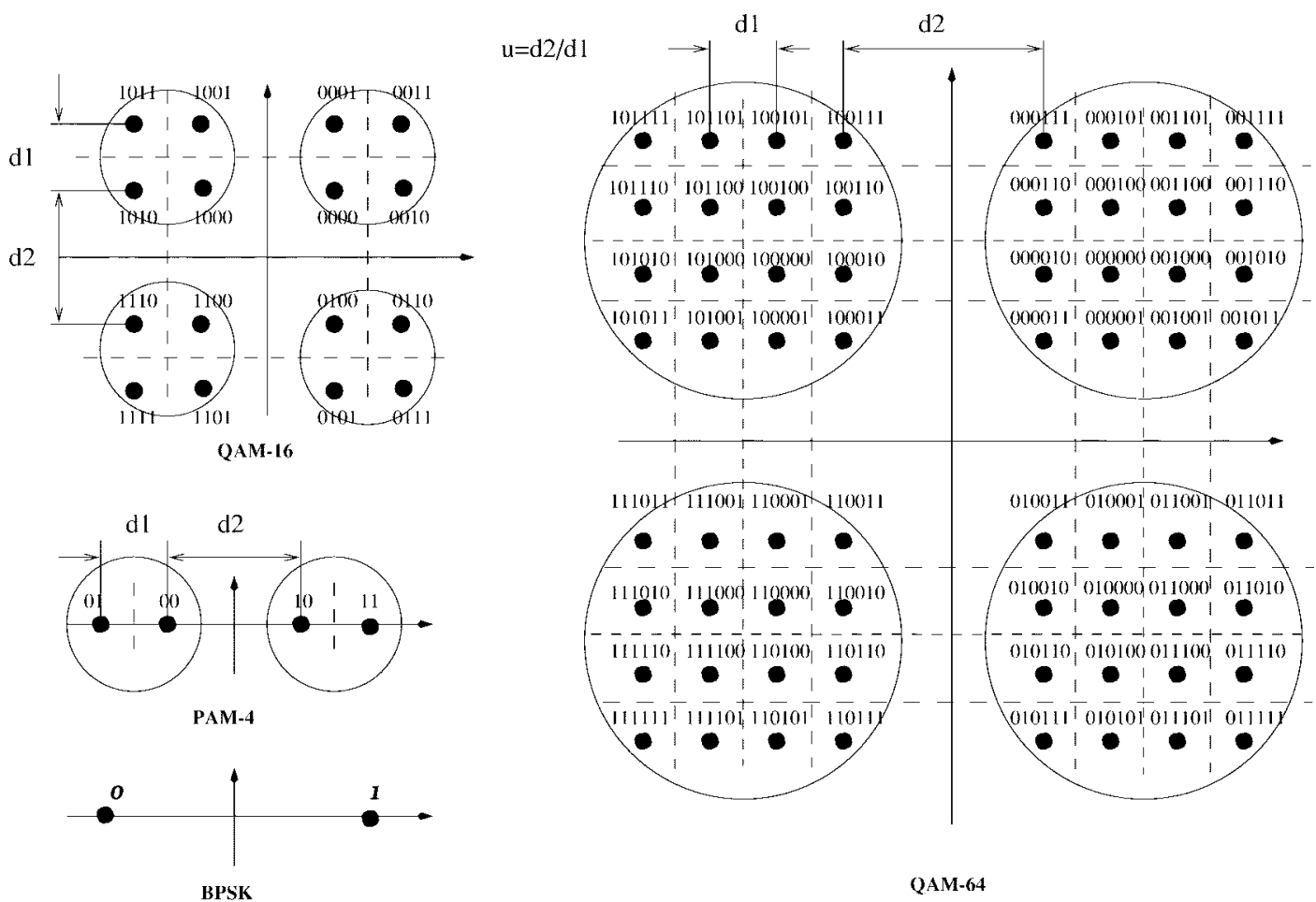

Fig. 2. Examples of multiresolution modulation constellation map.

modulation constellation of PAM-4, QAM-16, and QAM-64. PAM-4 is chosen over QAM-4 in order to provide UEP. The clouds are represented by the MSB's of the constellation points. For example, QAM-16 has binary representation of $\left(\begin{array}{llll}m_{3} & m_{2} & m_{1} & m_{0}\end{array}\right) .\left(m_{3}, m_{2}\right)$ represents the clouds and $\left(m_{1}, m_{0}\right)$ represents the satellites. $d 2$ is defined as "intracloud" distance, i.e., the minimum distance between two constellation points whose cloud bits are different, while $d 1$ represents "intercloud" distance or the distance between two neighboring constellation points sharing the same cloud bits. For given transmitted power and channel noise variance, $d 2$ and $d 1$ decide the error performance of the cloud and satellite bits. Different noise immunities are assigned to the information represented by clouds and satellites by allocating the transmitted power and adjusting the distance ratio $\mu=d 2 / d 1$. If a single source codeword is mapped to exactly one constellation point [6], the clouds will represent the coarse information (MSB's of this codeword) while the satellites represent the detailed information (LSB's of this codeword).

MR modulation provides an easy and effective way to adapt error performance of the information represented by different bits of a constellation. If source codewords are one-to-one mapped to a constellation, it is always true that information represented by clouds is of higher importance than that represented by satellites. However, in the proposed system, a single source codeword may be mapped to several constellation points, and therefore the above claim may fail. An example is shown in Fig. 3, where source rate $R_{s}=8$ and channel rate $R_{c}=2$, implying PAM-4. The source codeword is described as bit 7 (MSB) through bit 0 (LSB). Each constellation point of PAM-4 has a form of $\left(m_{1}, m_{0}\right)$,

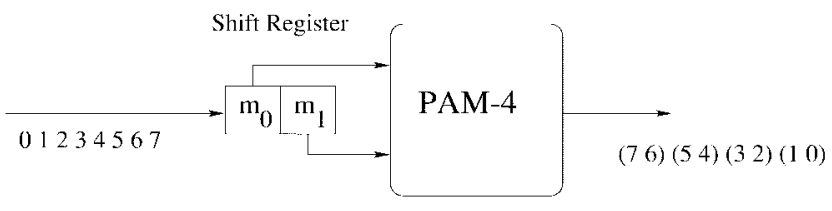

Direct Mapping

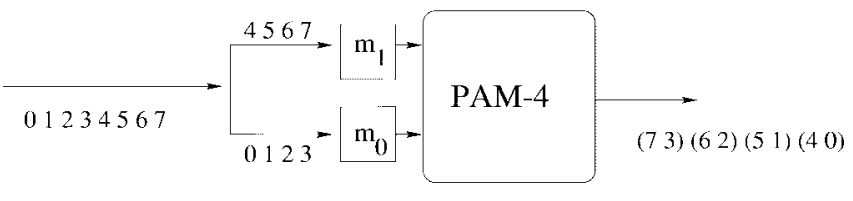

Bit Remapping

Fig. 3. Bit-mapping schemes.

where $m_{1}$ is the cloud and $m_{0}$ is the satellite. Therefore, the outputs of the modulator are $(7,6),(5,4),(3,2)$, and $(1,0)$. As such, bits 7, 5, 3, and 1 receive cloud-level error protection, and the remaining bits receive satellite-level error protection. But this is not appropriate, since bit 6 is more important than bits 5, 3, and 1 . The problem becomes worse if $R_{s}$ is not an integer multiple of $R_{c}$. Transmitting codewords of length $7\left(R_{s}=7\right)$ using PAM-4, the outputs become $(6,5),(4,3),(2,1),(0,6),(5,4)$, etc. Here, bit 0 from the first codeword is transmitted as the cloud bit and bit 6 from the next codeword as the satellite bit. Therefore, the transmitter has no control of which bit is transmitted as cloud or satellite. In the next section, we develop a new type of mapping scheme to remove such limitation. 


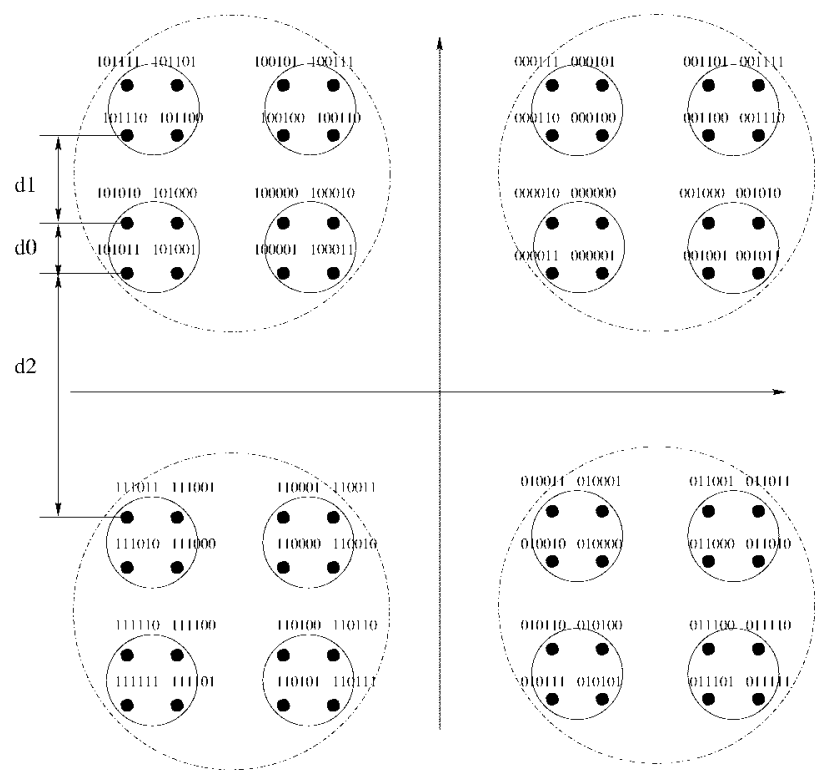

$\mathrm{ul}=\mathrm{d} 1 / \mathrm{d} 0, \mathrm{u} 2=\mathrm{d} 2 / \mathrm{d} 0$

OAM-64

Fig. 4. MR QAM-64 providing three levels of unequal error protection.

\section{B. Bit Remapping}

The objective of the mapping design is to minimize the channel distortion as follows:

$$
\begin{array}{ll}
\text { Given } & p\left(u \mid u^{\prime}\right), \quad u, u^{\prime} \in \Omega, x, x^{\prime} \in \mathcal{C} \\
\text { find } & F: \mathcal{C} \longrightarrow \Omega^{m}, \quad F(x)=\left\{u_{1}, u_{2}, \ldots, u_{m}\right\}
\end{array}
$$

which minimize the following

$$
\sum_{x, x^{\prime} \in \mathcal{C}} P\left(x^{\prime} \mid x\right) d\left(x^{\prime}, x\right)=\sum_{x, x^{\prime} \in \mathcal{C}} \prod_{i=1}^{m} p\left(u_{i}^{\prime} \mid u_{i}\right) d\left(x^{\prime}, x\right)
$$

where $\Omega$ represents the constellation set, $\Omega_{m}$ is the $m$ dimensional vector extension of $\Omega$, and $\mathcal{C}$ is the set of source codewords with cardinality of $2^{R_{i, s}}$.

Dynamic programming and simulated annealing can be used to find the optimal solution, which is difficult to find and computationally expensive. As shown in the above examples, bit order is essential in the design. We propose a simple bitremapping scheme that rearranges the bit order before sending to modulator [17]. This will map most MSB's of a codeword to clouds.

- Case I: $R_{s}<R_{c}$, multiple codewords are mapped to one constellation point.

$-R_{c}=m R_{s}$ for integer $m$.

Define $B_{i}^{j}$ as the $i$ th bit of codeword $j, i=$ $0 \cdots R_{s}, j=1 \cdots m$. The original bit order is

$$
\begin{aligned}
& \left(B_{R_{s}-1}^{1} \cdots B_{0}^{1} B_{R_{s}-1}^{2} B_{R_{s}-2}^{2} \cdots B_{R_{s}-1}^{k}\right. \\
& \left.B_{R_{s}-2}^{k} \cdots B_{R_{s}-1}^{m} \cdots B_{0}^{m}\right) .
\end{aligned}
$$

Rearrange to

$$
\begin{aligned}
& \left(B_{R_{s}-1}^{1} B_{R_{s}-1}^{2}, \ldots, B_{R_{s}-1}^{m} B_{R_{s}-2}^{1}\right. \\
& \left.\quad B_{R_{s}-2}^{2}, \ldots, B_{R_{s}-2}^{m}, \ldots, B_{0}^{1} B_{0}^{2}, \ldots, B_{0}^{m}\right) .
\end{aligned}
$$

This allows the clouds to carry as many codeword MSB's as possible.

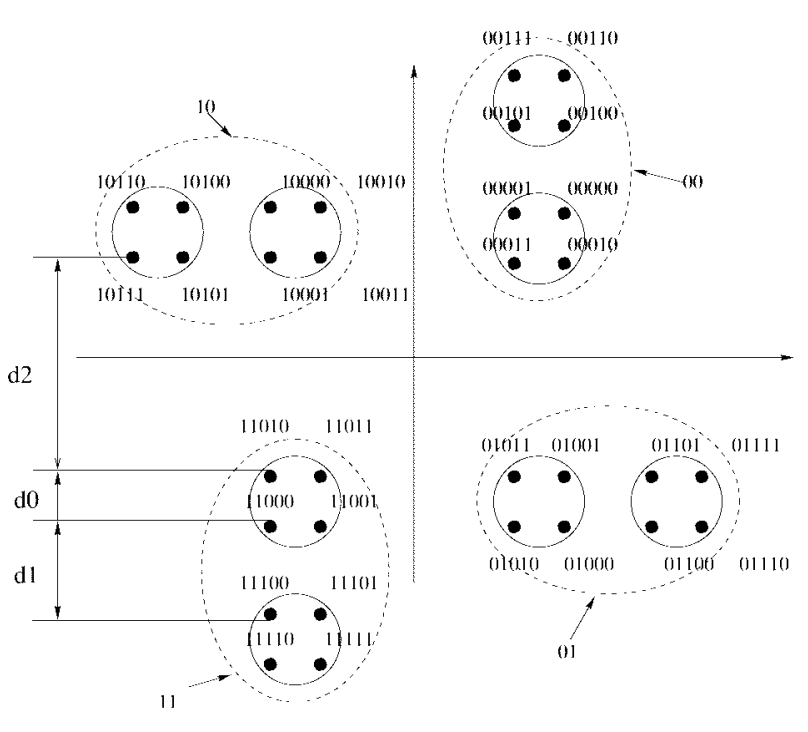

QAM32

$-R_{c}=m R_{s}+n$.

Transmit without rearrangement.

- Case II: $R_{s}>R_{c}$, one codeword is mapped to multiple constellation points.

$-R_{s}=m R_{c}$.

Divide source codeword consecutively to $R_{c}$ group, each of $m$ bits, i.e., assign bit $R_{s}-m$ to $R_{s}-2 m$ to group 1 and $R_{s}-2 m-1$ to $R_{s}-3 m$ to group 2, etc. Select bit $k$ from group 1 to $R_{c}$ and form the $k$ th modulation output. Define bit $i$ of group $j$ as $b_{i}^{j}$. The output constellation points are $\left(b_{0}^{1} b_{0}^{2} \cdots b_{0}^{R_{c}}\right),\left(b_{1}^{1} b_{1}^{2} \cdots b_{1}^{R_{c}}\right), \ldots$, $\left(b_{m}^{1} b_{m}^{2} \cdots b_{m}^{R_{c}}\right)$.

$-R_{s}=m R_{c}+n$.

Move $n$ LSB's to a temporary residue bit buffer and transmit the left $m R_{c}$ MSB's as a new codeword of length $R_{s}^{\prime}=m R_{c}$. Transmit the residue bits after completing the transmission of all the new codewords.

Fig. 3 illustrates our scheme and the conventional scheme. When the important bits' transmissions are delayed, or there is no time to transmit all the bits, the system can simply ignore the residue bits if there are any. As can be seen, the bit remapping changes the modulator outputs for $\left\{R_{s}=8, R_{c}=\right.$ $2\}$ to $(7,3),(6,2),(5,1)$, and $(4,0)$.

MR modulation proposed in [6] provides two levels of UEP in terms of clouds and satellites. This idea can be applied to the constellation recursively to produce multiple levels of UEP. MR QAM-64 providing three levels of UEP is shown in Fig. 4. The binary representation of the constellation points can be arranged such that the MSB's are always transmitted with equal or better error performance compared to the LSB's. Therefore, the proposed bit-remapping scheme can always map the MSB's of the codewords to the MSB's of the constellation points to achieve better protec- 


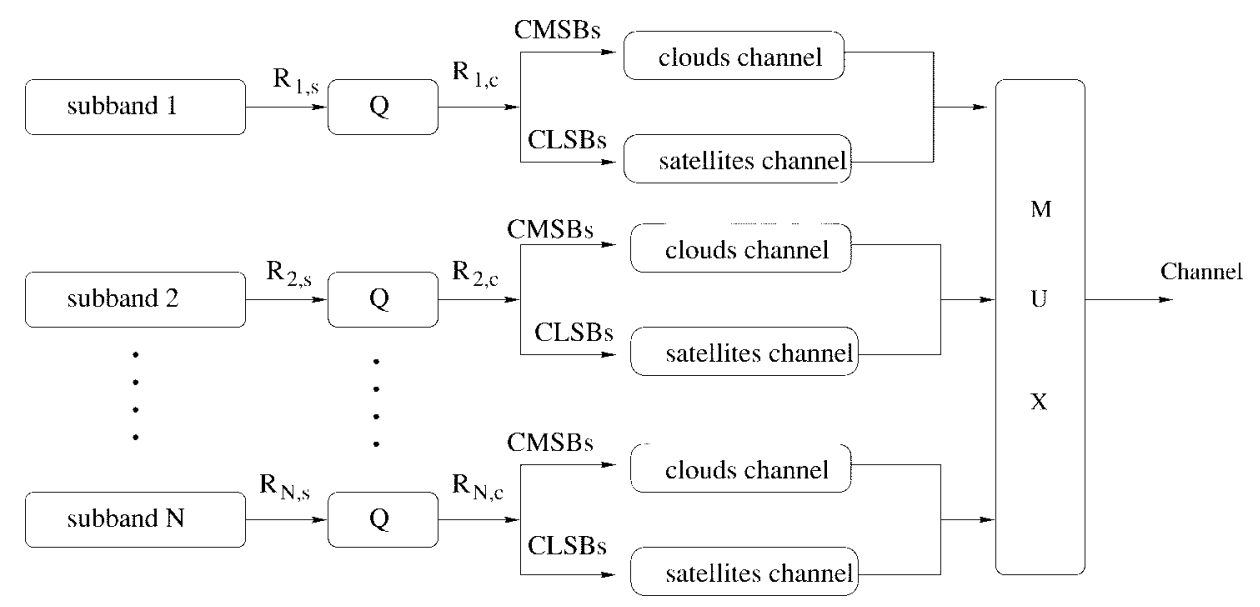

Fig. 5. Equivalent transmission system.

tion. It can be applied to all the modulation types, if the binary representations of the constellation point are arranged properly.

\section{Error Performance Computation}

The channel distortion defined in (2) relies on the computation of codeword error probability $P\left(a \mid b ; R_{c}, R_{s}\right)$. Since a single codeword is mapped to several constellation points if the source rate is a multiple of channel modulation rate, the codeword error probability is then a product of the symbol error probability. For example, if MR PAM-4 is employed

$$
\begin{aligned}
& P\left(a \mid b ; 2, R_{s}\right) \\
& \quad= \begin{cases}\prod_{i=0}^{k-1} p_{s}^{2}\left(\left(a_{2 k-2 i-1} a_{2 k-2 i-2}\right) \mid\right. & R_{s}=2 k \\
\prod_{i=0}^{k-1} p_{s}^{2}\left(\left(b_{2 k-2 i-1} b_{2 k-2 i-2}\right)\right) & \end{cases}
\end{aligned}
$$

where $a_{i}$ is the $i$ th bit of codeword $a$. And

$$
p_{b}^{2}\left(a_{0} \mid b_{0}\right)= \begin{cases}p_{b}^{2}\left(\frac{E}{N_{0}}\right), & a_{0} \neq b_{0} \\ 1-p_{b}^{2}\left(\frac{E}{N_{0}}\right), & a_{0}=b_{0}\end{cases}
$$

where $p_{b}^{2}\left(E / N_{0}\right)$ is the averaged bit error probability of PAM4 and $p_{s}^{2}($.$) is the symbol error probability of PAM-4. When$ $R_{s}$ is an odd number, there is a residue bit $a_{0}$, the LSB of codeword $a$. It is transmitted separately to ensure the MSB's of the codeword being mapped to the clouds.
In general, $P\left(a \mid b ; R_{c}, R_{s}\right)$ is computed as

$$
\begin{aligned}
& P\left(a \mid b ; R_{c}, R_{s}\right) \\
& =\left\{\begin{array}{l}
\prod_{i=0}^{m} p_{s}^{R_{c}}\left(F^{R_{s}, R_{c}}(a)_{i} \mid F^{R_{s}, R_{c}}(b)_{i}\right) \\
R_{s}=m \cdot R_{c} \\
\prod_{i=0}^{m} p_{s}^{R_{c}}\left(F^{R_{s}, R_{c}}(a)_{i} \mid F^{R_{s}, R_{c}}(b)_{i}\right) \\
\cdot p_{R_{c}}\left(F^{R_{s}, R_{c}}(a)_{m+1} \mid F^{R_{s}, R_{c}}(b)_{m+1}\right) \\
R_{s}=m \cdot R_{c}+n
\end{array}\right.
\end{aligned}
$$

where $F^{R_{s}, R_{c}}(a)_{i}$ is the $i$ th constellation point that codeword $a$ being mapped to and $F^{R_{s}, R_{c}}(a)_{m+1}$ represents the $n$ residue bits. We define

$$
p_{R_{c}}(x \mid y)=\left(p_{b}^{R_{c}}\right)^{l}\left(1-p_{b}^{R_{c}}\right)^{n-l}
$$

if $x, y$ differ in $l$ bits.

In such a way, $P\left(a \mid b ; R_{c}, R_{s}\right)$ cannot be represented as a close function of power $E$, channel modulation rate $R_{c}$, or source rate $R_{s}$. Therefore, the optimization has to compute the $P\left(a \mid b ; R_{c}, R_{s}\right)$ for all the codewords in order to derive the corresponding channel distortion. To seek the best $R_{c}$ and $R_{s}$, integer programming can be used. However, power allocation cannot be achieved. Therefore, in the simulation, we distribute the transmitted power evenly on all the subbands to be transmitted.

\section{Simplified Distortion ANALYSIS}

To compute the channel distortion defined in (2), we have to go through all possible codewords and compute the symbol error probabilities, which requires extensive computations. In this section, we will show that by applying bit remapping, the channel distortion can be approximated as the sum of the distortion caused by corrupted satellite bits and cloud bits. This will not only decrease the distortion computations dramatically but also allow the power allocation to be employed to further improve the performance.

After applying bit remapping, the bits of a source codeword are mapped to cloud bits and satellite bits. We classify the 

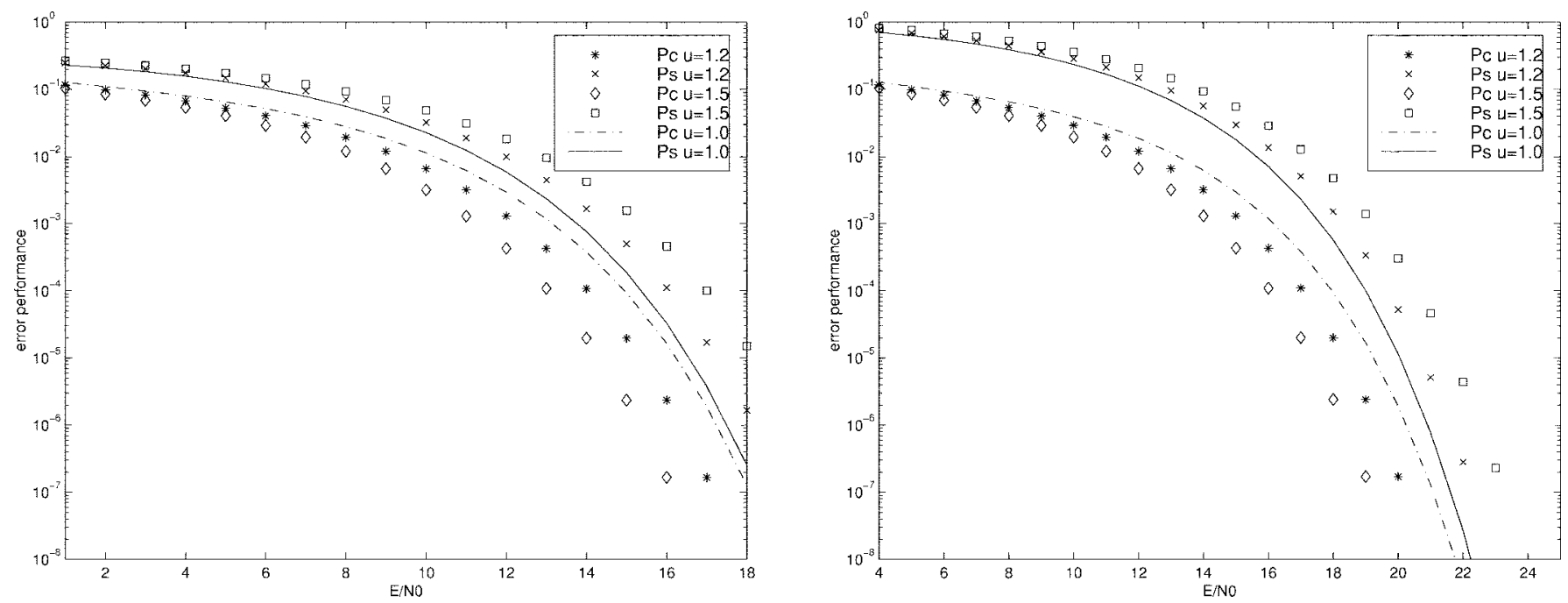

Fig. 6. Cloud and satellite error-performance comparison.
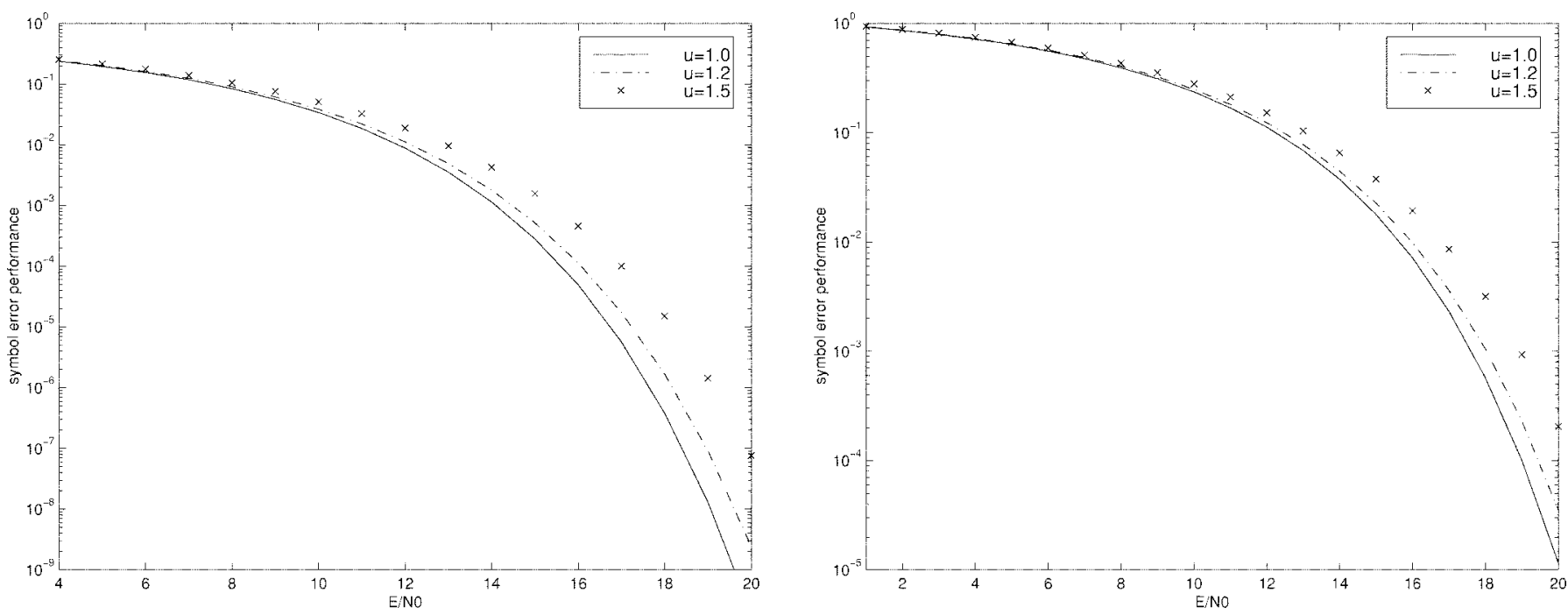

Fig. 7. Symbol error-performance comparison.

residue bits to satellite bits. For codewords of length $R_{i, s}$, the number of information bits carried by the clouds in an MR modulation of rate $R_{i, c}$ is computed as $C_{\left(R_{i, c}, R_{i, s}\right)}$

$$
C_{\left(R_{i, s}, R_{i, c}\right)}= \begin{cases}0, & R_{i, c}>R_{i, s} \\ 0, & \text { BPSK } \\ \frac{R_{i, s}}{R_{i, c}}, & \text { xPAM } \\ \frac{2 R_{i, s}}{R_{i, c}}, & \text { xQAM. }\end{cases}
$$

In other words, $C_{\left(R_{i, c}, R_{i, s}\right)}$ MSB's (referred to as CMSB's below) of each codeword are mapped to the clouds and $R_{i, s}-C_{\left(R_{i, c}, R_{i, s}\right)}$ LSB's (referred to as CLSB's below) to the satellites. It is equivalent to transmit these two types of bits through different subchannels. Fig. 5 shows the equivalent transmission systems. It was shown in [18] that different transmitted power and bit rate can be assigned to the subchannels to achieve unequal error protection. We define $P^{c}\left(R_{i, c}, E\right)$ as the probability of the received cloud bits' being corrupted.
Similarly, $P^{s}\left(R_{i, c}\right)$ represents the probability of receiving erroneous satellites and $P^{\mathrm{sc}}\left(R_{i, c}\right)$ represents the probability of both the received clouds and satellites' being corrupted. Assuming that $P^{\mathrm{sc}}\left(R_{i, c}\right)$ is negligible compared to the others; (2) can be approximated as

$$
\begin{aligned}
D_{i, c}\left(R_{i, s}, R_{i, c}\right) & \\
\approx \sum_{n=0}^{N_{i, s}-1}\{ & \sum_{m=1}^{C_{\left(R_{i, c}, R_{i, s}\right)}} \sum_{k=0}^{N_{i, s}-1} \delta_{m}^{c}\left(x_{n}, x_{k}\right) P^{c}\left(R_{i, c}, E\right)^{k} \\
& \cdot\left(1-P^{c}\left(R_{i, c}, E\right)\right)^{C_{\left(R_{i, s}, R_{i, c}\right)}-k} D\left(x_{n}, x_{k}\right) \\
& +\sum_{m=1}^{R_{i, s}-C_{\left(R_{i, c}, R_{i, s}\right)}} \sum_{k=0}^{N_{i, s}-1} \delta_{m}^{s}\left(x_{n}, x_{k}\right) \\
& \cdot P^{s}\left(R_{i, c}, E\right)^{k} \\
& \cdot\left(1-P^{s}\left(R_{i, c}, E\right)\right)^{R_{i, s}-C_{\left(R_{i, s}, R_{i, c}\right)}-k} \\
& \left.\cdot D\left(x_{n}, x_{k}\right)\right\}
\end{aligned}
$$






(a)

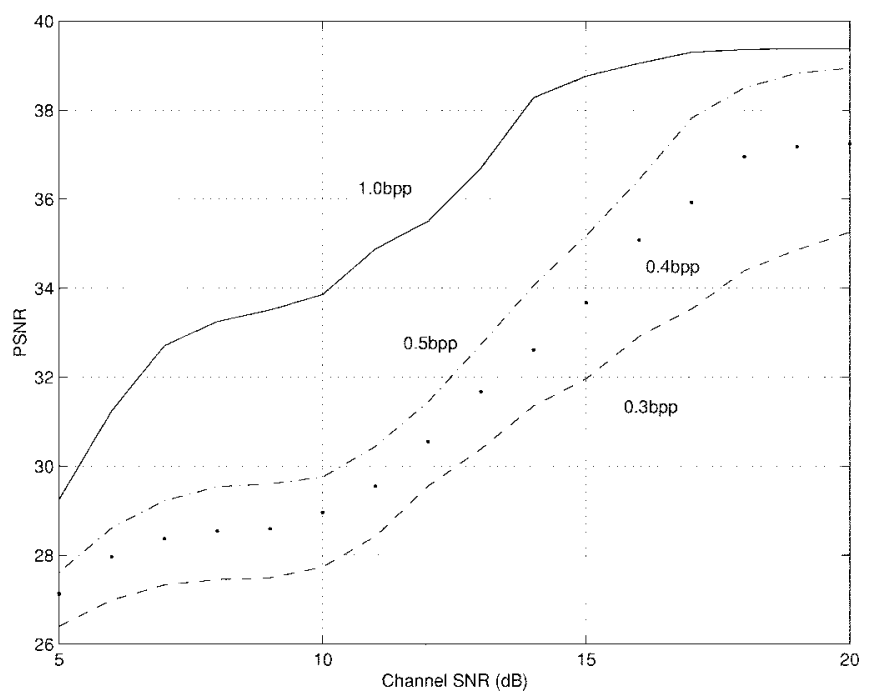

(b)

Fig. 8. PSNR versus SNR performance for frame 3 of Salesman sequence using (a) Method A with $\mu=2.0$ and (b) Method B with $\mu=1.2$.
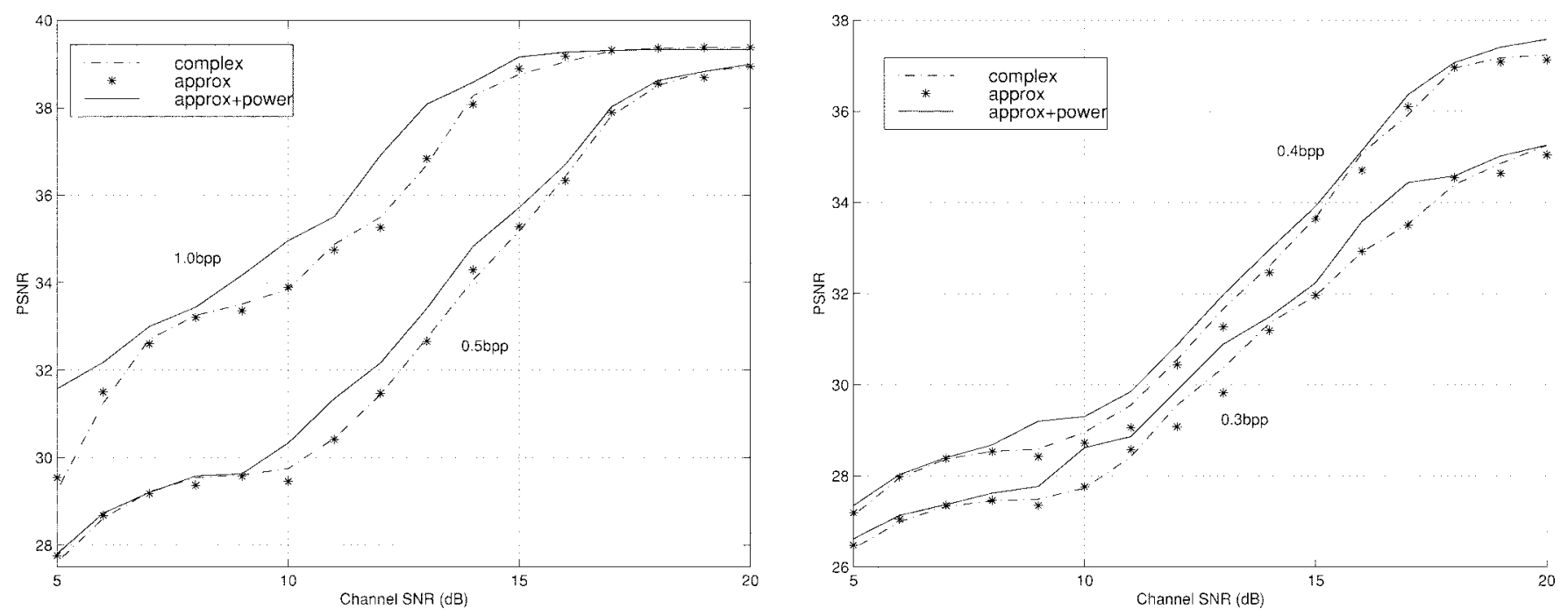

Fig. 9. PSNR versus SNR performance comparison of complex and approximation approach.

where

$$
\begin{aligned}
& \delta_{m}^{c}\left(x_{n}, x_{k}\right) \\
& \quad= \begin{cases}1, & \text { if } Q_{R_{i, s}}\left(x_{n}\right), Q_{R_{i, s}}\left(x_{k}\right) \text { differ in } m \text { CMSB's } \\
0, & \text { otherwise }\end{cases} \\
& \delta_{m}^{s}\left(x_{n}, x_{k}\right) \\
& \quad= \begin{cases}1, & \text { if } Q_{R_{i, s}}\left(x_{n}\right), Q_{R_{i, s}}\left(x_{k}\right) \text { differ in } m \text { LMSB's } \\
0, & \text { otherwise. }\end{cases}
\end{aligned}
$$

\section{A. Cloud and Satellite Error Performance}

We will compute cloud and satellite error probability in this section. We also provide numerical comparisons between the MR modulation and the commonly used modulation. The latter is a special case of the MR modulation where $\mu=1$. Although $\mu \neq 1$ is not optimal in terms of averaged symbol error probability, it can provide unequal error protection flexibly. Assuming transmittal of constellation point 00 using PAM-4, from Fig. 2, we can compute the symbol error probability as follows:

$$
\begin{aligned}
& p_{s}(01 \mid 00)=Q\left(\frac{\mu d}{\sqrt{2 N_{0}}}\right) \\
& p_{s}(10 \mid 00)=Q\left(\frac{\mu d}{\sqrt{2 N_{0}}}\right)-Q\left(\frac{(2 \mu+1) d}{\sqrt{2 N_{0}}}\right) \\
& p_{s}(11 \mid 00)=Q\left(\frac{(2 \mu+1) d}{\sqrt{2 N_{0}}}\right) .
\end{aligned}
$$

We represent $d 1$ and $d 2$ as $d$ and $\mu d$, respectively. Therefore, the probability of a cloud bit's being corrupted given transmittal of point 00 is approximately $p_{s}(10 \mid 00)+p_{s}(11 \mid 00)=$ $Q\left(\mu d / \sqrt{2 N_{0}}\right)$. The average error probability of the cloud bit is

$$
p^{c} \approx \frac{1}{2} Q\left(\frac{\mu d}{\sqrt{2 N_{0}}}\right)
$$




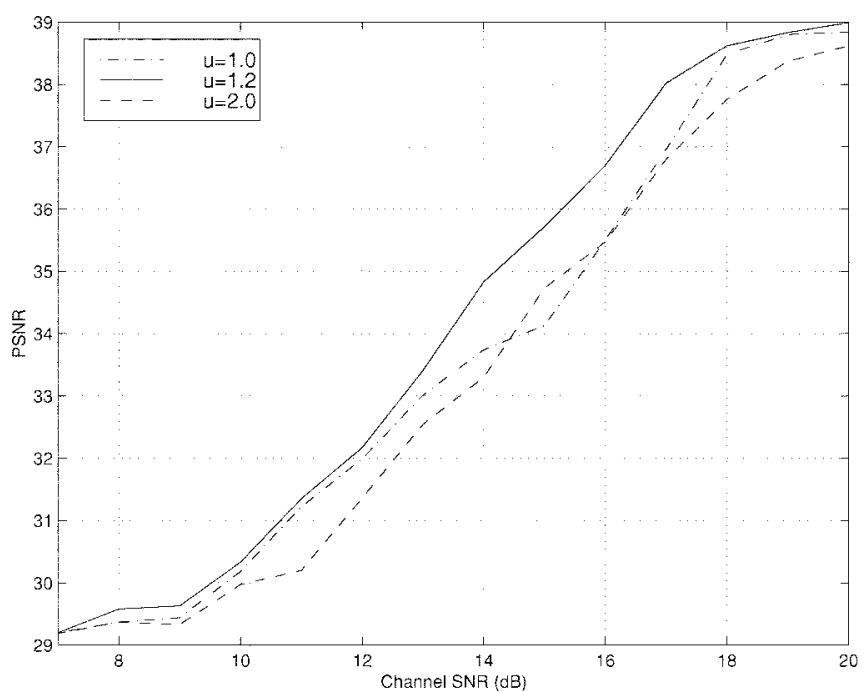

(a)

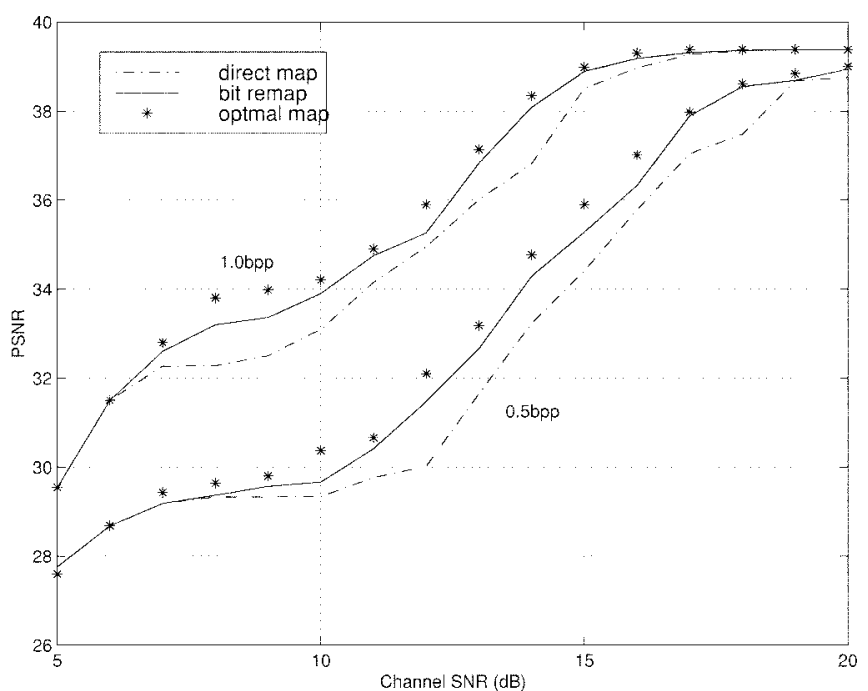

(b)

Fig. 10. (a) PSNR versus SNR performance of Salesman sequence frame 3 coded using different $\mu$ values, where $\mu=1.0$ stands for the common or non-MR constellation scheme. (b) For same image, PSNR versus SNR performance comparison of the systems without mapping, with intelligent bit remapping and with optimal mapping.

The average error probability of the satellite bit is

$$
p^{s} \leq Q\left(\frac{d}{\sqrt{2 N_{0}}}\right)-\frac{1}{2} Q\left(\frac{(\mu+2) d}{\sqrt{2 N_{0}}}\right)<Q\left(\frac{d}{\sqrt{2 N_{0}}}\right) \text {. }
$$

Similarly, for QAM-16

$$
\begin{aligned}
& p^{c} \approx Q\left(\frac{\mu d}{\sqrt{2 N_{0}}}\right) \\
& p^{s} \leq 2 Q\left(\frac{d}{\sqrt{2 N_{0}}}\right) .
\end{aligned}
$$

In general, for average symbol energy $E$, we can represent the BER of clouds and satellites as shown in (19) and (20) at the bottom of the page. $K_{R_{i, c}}^{s}($.$) and K_{R_{i, c}}^{c}($.$) are the average$ number of nearest neighbors in terms of satellite bits and cloud bits, respectively.

For XPAM modulations, there are $L=2^{R_{i, c}-1}$ constellation points in each direction. Among $L$ points, $L-2$ points have two nearest neighbors in terms of satellite bits and two points only have one; therefore

$$
\begin{aligned}
K_{R_{i, c}}^{s} & =\frac{2(L-2)+2}{L} \\
& =2-4 \cdot 2^{-R_{i, c}} .
\end{aligned}
$$

For xQAM, assume that in each $I$ and $Q$ direction, the number of points is $L_{I}$ and $L_{Q}$, respectively. There are $\left(L_{I}-2\right)\left(L_{Q}-2\right)$ points with four nearest neighbors in terms

$$
\begin{aligned}
& P^{c}\left(R_{i, c}, E\right) \approx\left\{\begin{array}{l}
0, \quad R_{i, c}=1 \\
K_{R_{i, c}}^{c} Q\left(\frac{\mu d}{\sqrt{2 N_{0}}}\right)=K_{R_{i, c}}^{c} Q\left(\mu \theta_{R_{i, c}}^{\mu} \sqrt{\frac{E}{2 N_{0}}}\right), \quad R_{i, c}>1
\end{array}\right. \\
& P^{s}\left(R_{i, c}, E\right) \approx\left\{\begin{array}{l}
Q\left(\frac{d}{\sqrt{2 N_{0}}}\right)=Q\left(2 \sqrt{\frac{E}{2 N_{0}}}\right), \quad R_{i, c}=1 \\
K_{R_{i, c}}^{s} Q\left(\frac{d}{\sqrt{2 N_{0}}}\right)=K_{R_{i, c}}^{s} Q\left(\theta_{R_{i, c}}^{\mu} \sqrt{\frac{E}{2 N_{0}}}\right), \quad R_{i, c}>1
\end{array}\right.
\end{aligned}
$$

where

$$
\theta_{R_{i, c}}^{\mu}=\left\{\begin{array}{l}
\sqrt{\frac{1}{\left(\mu+2^{R_{i, c}-1}-1\right)^{2}+\frac{2^{2 R_{i, c}-2}-1}{3}},}, \text { PAM } \\
\sqrt{\frac{2}{\left(\mu+2^{R_{i, c}-2 / 2-1}\right)^{2}+\frac{2^{R_{i, c}-2}-1}{3}},} \text { QAM and even } R_{i, c} \\
\sqrt{\frac{2}{\left.\mu+3 \cdot 2^{R_{i, c}-3 / 2}-2\right)^{2}+\frac{13 \cdot 2^{R_{i, c}-3}-4}{12}},} \text { QAM and odd } R_{i, c}
\end{array}\right.
$$




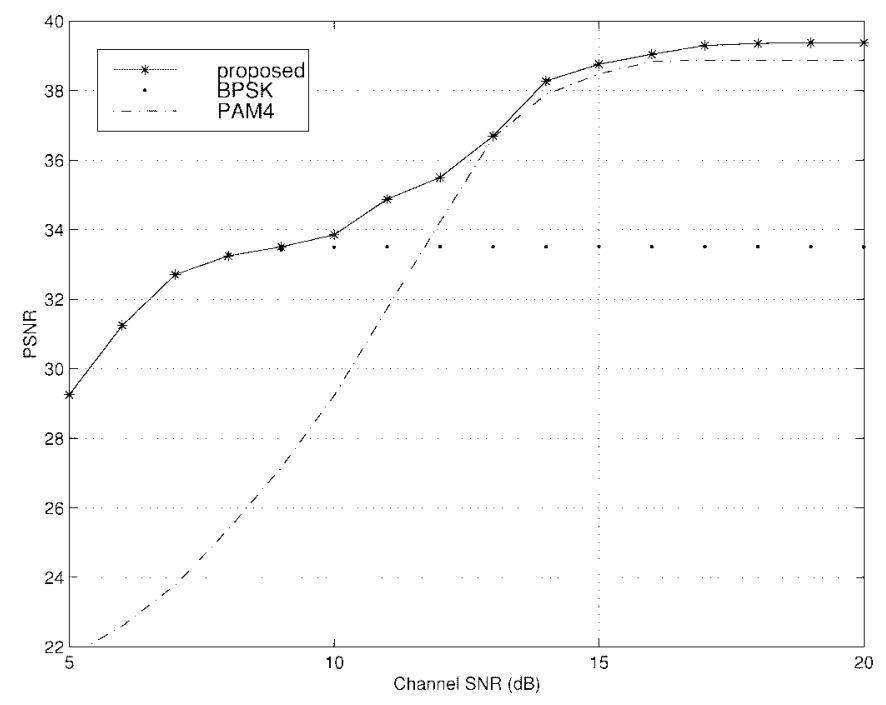

(a)

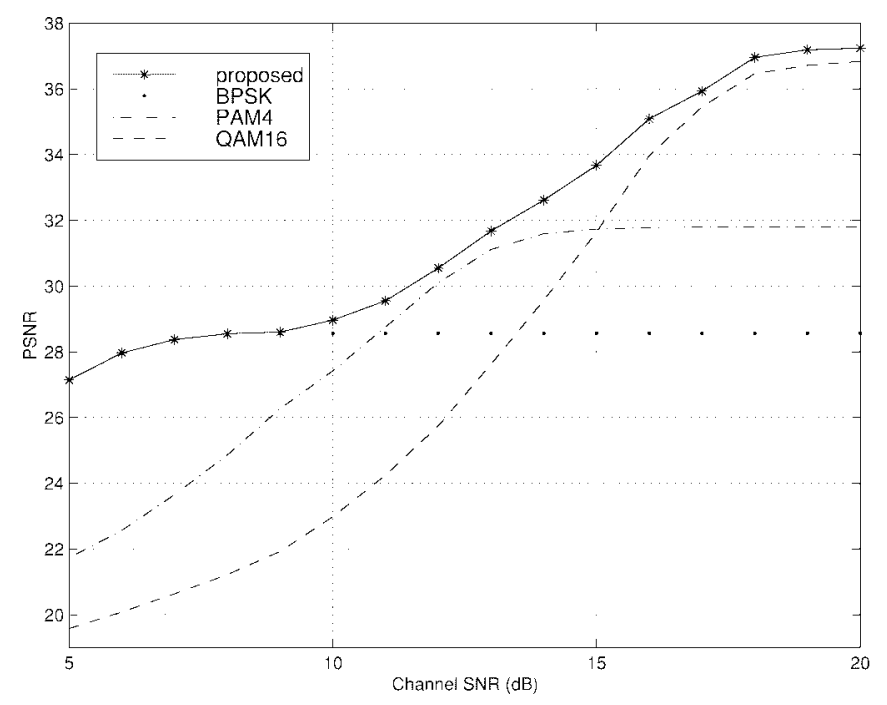

(c)

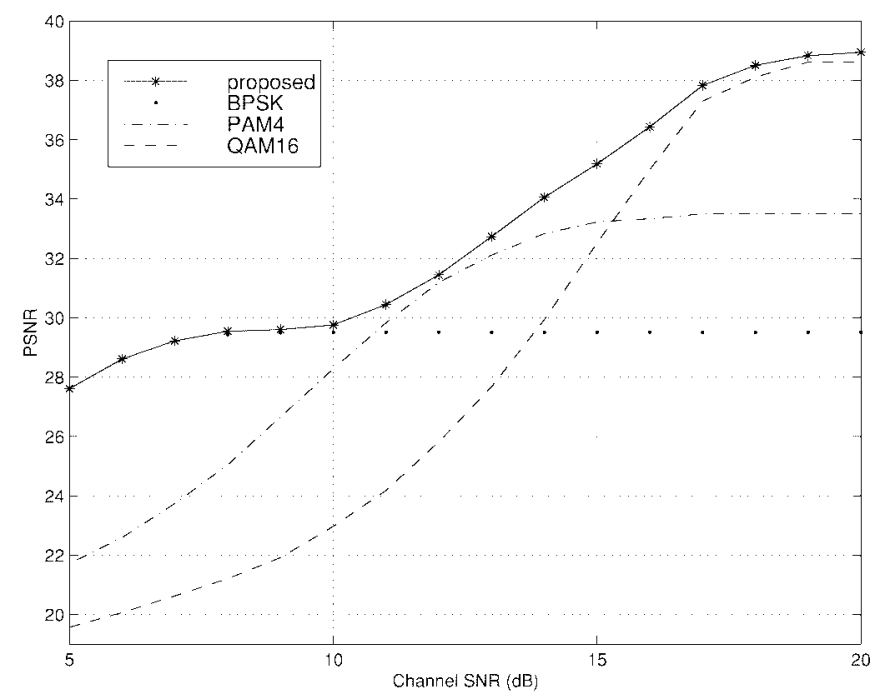

(b)

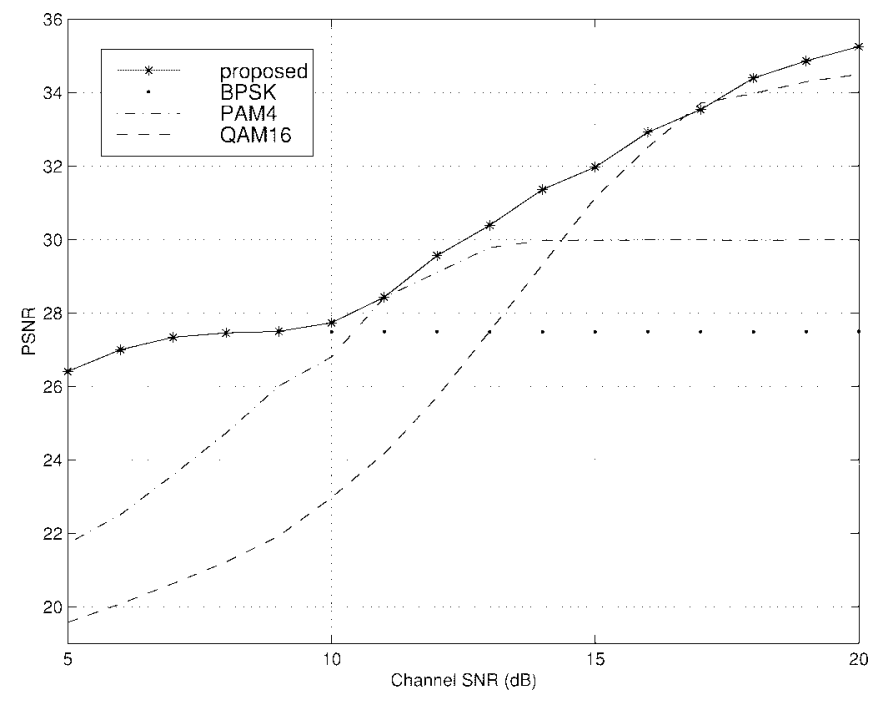

(d)

Fig. 11. PSNR versus SNR performance comparison of different systems for Salesman image using Method B at (a) 1.0, (b) 0.5, (c) 0.4, and (d) 0.3 bpp.

of satellites, $2\left(L_{I}-2\right)+2\left(L_{Q}-2\right)$ points with three neighbors, and four points with two neighbors. It follows that

$$
\begin{aligned}
& K_{R_{i, c}}^{s} \\
& \quad=\frac{4\left(L_{I}-2\right)\left(L_{Q}-2\right)+3\left(2\left(L_{I}-2\right)+2\left(L_{Q}-2\right)\right)+4}{L_{I} L_{Q}} \\
& \quad=4-2\left(\frac{1}{L_{I}}+\frac{1}{L_{Q}}\right) .
\end{aligned}
$$

If $R_{i, c}$ is an even integer, $L_{I}=L_{Q}=2^{\left(R_{i, c}-1\right) / 2}$. If $R_{i, c}$ is odd, $L_{I}=L_{Q} / 2=2^{\left(R_{i, c}-3\right) / 2}$. Upon this, we get

$$
K_{R_{i, c}}^{s}= \begin{cases}1, & \text { BPSK } \\ 2-4 \cdot 2^{-R_{i, c},} & \text { PAM } \\ 4-8 \cdot 2^{-\left(R_{i, c} / 2\right)}, & \text { QAM with even } R_{i, c} \\ 4-6 \cdot 2^{-\left(\left(R_{i, c}-1\right) / 2\right)}, & \text { QAM with odd } R_{i, c}\end{cases}
$$

while $K_{R_{i, c}}^{c}$ is approximated similarly

$$
K_{R_{i, c}}^{c} \approx \begin{cases}0, & \text { BPSK } \\ 2 \cdot 2^{-R_{i, c}}, & \text { PAM } \\ 4 \cdot 2^{-\left(R_{i, c} / 2\right)}, & \text { QAM with even } R_{i, c} \\ 3 \cdot 2^{-\left(\left(R_{i, c}-1\right) / 2\right)}, & \text { QAM with odd } R_{i, c} .\end{cases}
$$

We compare the cloud and satellite error performance at different $\mu$, as shown in Fig. 6. The averaged symbol error probability is sketched in Fig. 7. As can be seen, the error-performance difference between the clouds and satellites increases as $\mu$ increases. Clouds receive higher protection by trading off that of satellites. As a result, the averaged symbol error performance degrades.

\section{B. Distortion Approximation}

The above approximations can be used to simplify the optimization problem. Making the assumption that $P^{c}($.$) and$ 


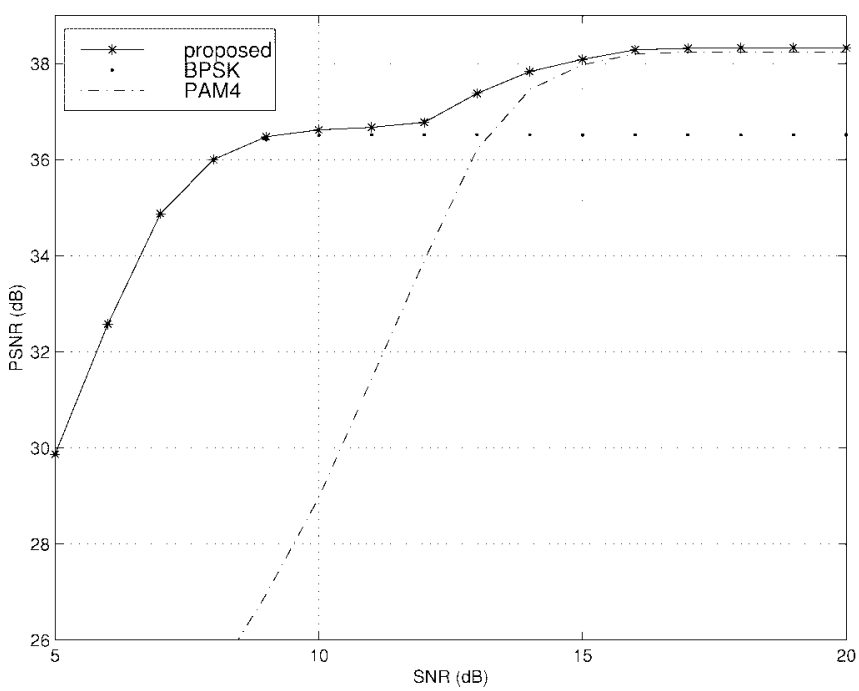

(a)

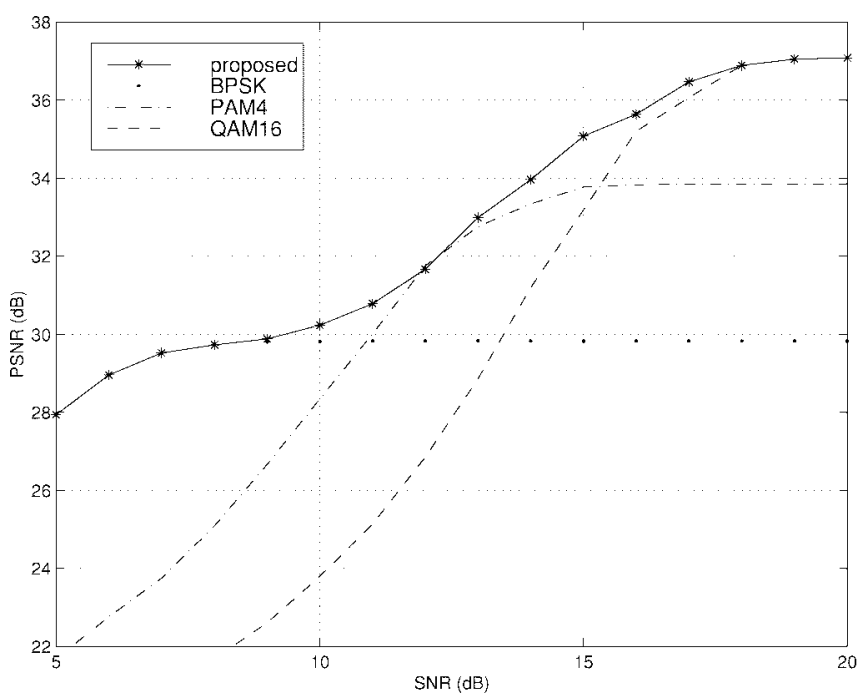

(c)



(b)

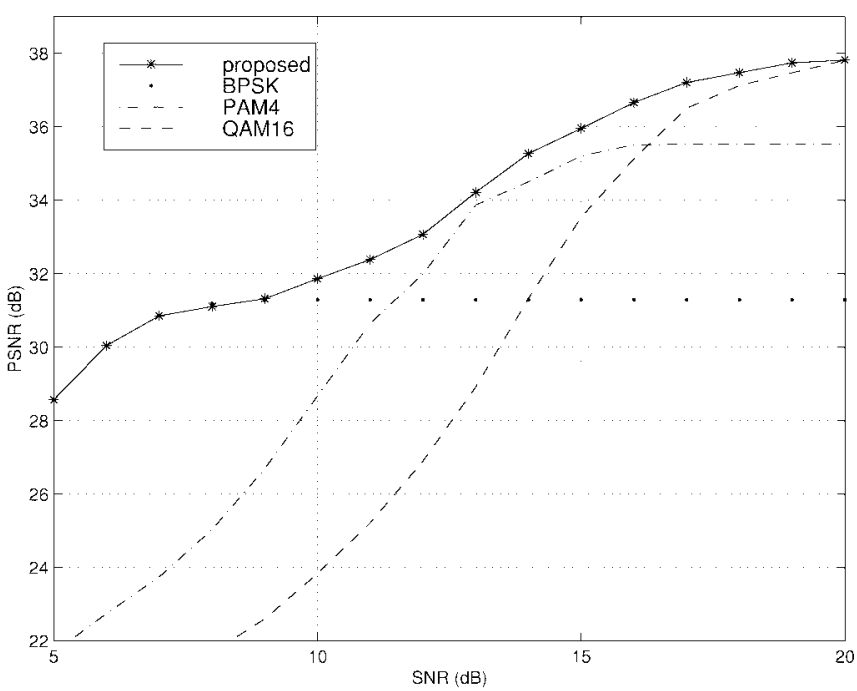

(d)

Fig. 12. PSNR versus SNR performance comparison of different systems for Salesman video sequence using Method B at (a) 1.0, (b) 0.5, (c) 0.4, and (d) 0.3 bpp.

$P^{s}($.$) are small enough such that$

$$
\begin{aligned}
P^{c}(.)^{k} \approx 0 & \text { and } P^{s}(.)^{k} \approx 0 \\
\left(1-P^{c}(.)\right)^{k} \approx 1 & \text { and }\left(1-P^{s}(.)\right)^{k} \approx 1 \quad k>1
\end{aligned}
$$

we reduce (13) into

$$
\begin{aligned}
D_{i, c} & \left(R_{i, s}, R_{i, c}, E\right) \\
\approx & \sum_{n=0}^{N_{i, s}-1} \sum_{k=0}^{N_{i, s}-1} \delta_{1}^{c}\left(x_{n}, x_{k}\right) P^{c}\left(R_{i, c}, E\right) D\left(x_{n}, x_{k}\right) \\
& +\sum_{n=0}^{N_{i, s}-1} \sum_{k=0}^{N_{i, s}-1} \delta_{1}^{s}\left(x_{n}, x_{k}\right) P^{s}\left(R_{i, c}, E\right) D\left(x_{n}, x_{k}\right) \\
= & W^{c}\left(R_{i, s}, R_{i, c}\right) P^{c}\left(R_{i, c}, E\right) \\
& +W^{s}\left(R_{i, s}, R_{i, c}\right) P^{s}\left(R_{i, c}, E\right)
\end{aligned}
$$

where $W^{c}($.$) is the averaged distortion of a single erroneous$ source bit mapped to cloud and $W^{s}($.$) is the averaged distor-$ tion if error happens to a codeword bit mapped to satellite.
This simplification removes most symbol error-probability computations. In addition, it allows the system to allocate the transmitted power efficiently to the subbands rather than using an equal amount of power. The optimization is defined as

$$
\begin{aligned}
\operatorname{Min} D_{\text {total }}= & \sum_{i=1}^{N} D_{i, s}\left(R_{i, s}\right)+W^{c}\left(R_{i, s}, R_{i, c}\right) \\
& \cdot K_{R_{i, c}^{c}}^{c} Q\left(\mu \theta_{R_{i, c}}^{\mu} \sqrt{\frac{E_{i} / l_{i}}{2 N_{0}}}\right) \\
& +W^{s}\left(R_{i, s}, R_{i, c}\right) K_{R_{i, c}^{s}}^{s} Q\left(\theta_{R_{i, c}}^{\mu} \sqrt{\frac{E_{i} / l_{i}}{2 N_{0}}}\right)
\end{aligned}
$$

subject to

$$
\sum_{i=1}^{N} l_{i}=R_{\text {budget }}, \sum_{i=1}^{N} E_{i} \leq E_{T} \cdot R_{\text {budget }}
$$




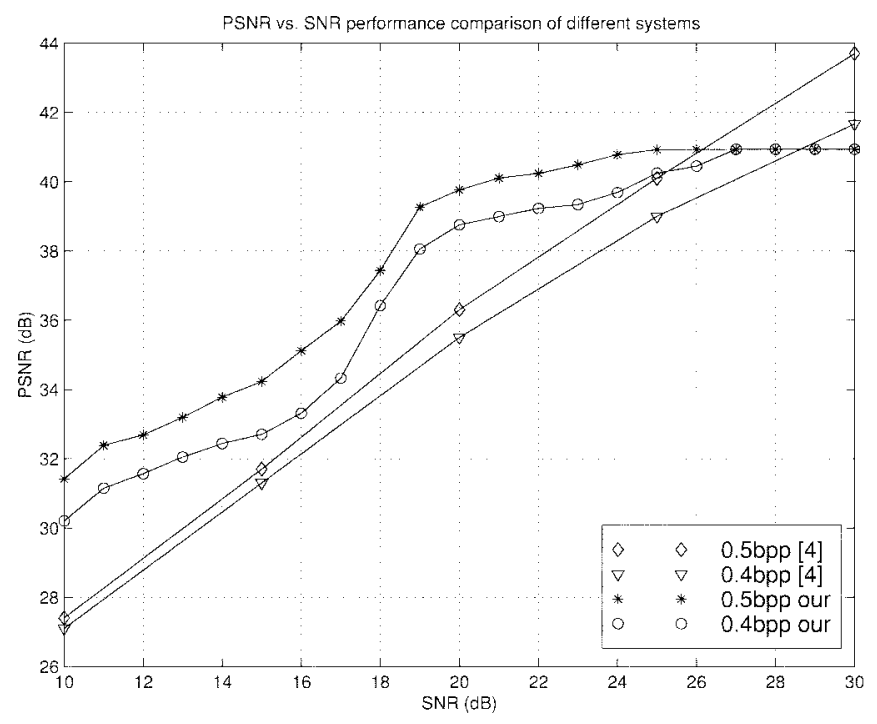

(a)

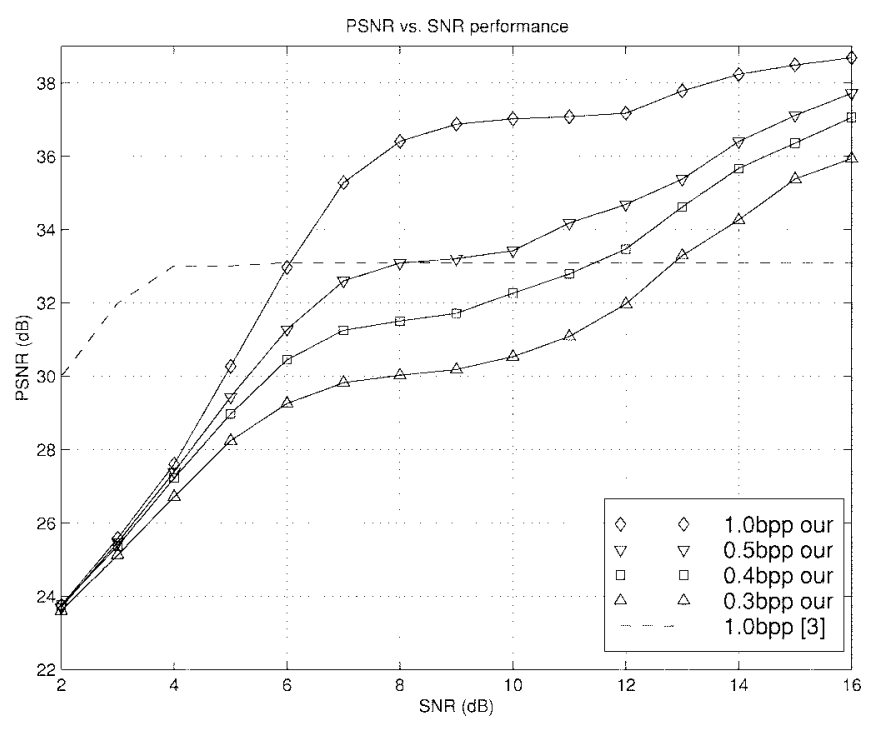

(b)

Fig. 13. (a) PSNR versus SNR performance comparison of proposed system and intelligent mapping system at different rates. (b) PSNR versus SNR performance comparison of proposed system $(1.0,0.5,0.4,0.3 \mathrm{bpp})$ and joint source and channel coding system (1.0 bpp).

where $E_{T}$ is the averaged transmitted power. $E_{i} / l_{i}$ is the average transmitted power per constellation point, with

$$
l_{i}=\frac{S_{i} \cdot R_{i, s}}{d_{i} \cdot R_{i, c}}
$$

where $S_{i}$ and $d_{i}$ represent the size and VQ dimension of subband $i$, respectively.

The optimization is solved by applying a Lagrange multiplier $\vec{\lambda}=\left(\lambda_{1}, \lambda_{2}\right)$. For a given $\left(\lambda_{1}, \lambda_{2}\right)$, it is turned into the optimization for each subband

$$
\operatorname{Min} W_{i}(\vec{\lambda})=D_{i, s}+D_{i, c}+\lambda_{1} l_{i}+\lambda_{2} E_{i}
$$

Differentiating with respect to $E_{i}$, we get

$$
\begin{gathered}
W^{c}\left(R_{i, s}, R_{i, c}\right) K_{R_{i, c}}^{c} \frac{\mu \theta_{R_{i, c}}^{\mu}}{\sqrt{2 E_{i} l_{i} N_{0}}} \\
\cdot \exp \left(-\frac{E_{i}\left(\mu \theta_{R_{i, c}}^{\mu}\right)^{2}}{\left.4 l_{i} N_{0}\right)}\right)+W^{s}\left(R_{i, s}, R_{i, c}\right) \\
\cdot K_{R_{i, c}}^{s} \frac{\theta_{R_{i, c}}^{\mu}}{\sqrt{2 E_{i} l_{i} N_{0}}} \exp \left(-\frac{E_{i}\left(\theta_{R_{i, c}}^{\mu}\right)^{2}}{\left.4 l_{i} N_{0}\right)}\right)=\lambda_{2} .
\end{gathered}
$$

Define

$$
\begin{aligned}
& \Phi_{\left\{\alpha_{1}, \alpha_{2}, \beta_{1}, \beta_{2}\right\}}(\omega) \\
& \quad=\sqrt{\frac{1}{2 \omega}}\left[\alpha_{1} \beta_{1} \exp \left(-\frac{\beta_{1}^{2} \omega}{4}\right)+\alpha_{2} \beta_{2} \exp \left(-\frac{\beta_{2}^{2} \omega}{4}\right)\right]
\end{aligned}
$$

which is monotonic for $\omega>0$. For a given $\lambda_{2}$, we have (31), shown at the bottom of the page, which is a monotonic function of $\lambda_{2}>0$. The optimal $\lambda_{2}$ satisfies

$$
\sum_{i=1}^{N} E_{i}\left(\lambda_{2}\right) \leq E_{T} \cdot B_{\text {budget }} .
$$

The optimization consists of three levels. The first level finds the optimal $\left\{R_{i, c}, R_{i, s}\right\}_{i=1}^{N}$ and $E_{i}\left(R_{i, c}, R_{i, s}, \lambda_{2}\right)$, in terms of minimizing (28), for given $\lambda_{1}$ and $\lambda_{2}$. The second level seeks the $\lambda_{1}$ that yields minimum $D_{\text {total }}$ and satisfies data bit constraints for a given $\lambda_{2}$. The third level finds the optimal $\lambda_{2}$ in terms of transmitted power constraint $\sum_{i=1} N \leq$ $E_{T} B_{\text {budget }}$.

\section{Simulation Results IN AWGN ChanNel}

In the simulation, we use a subband-based encoder operating in "intraframe only" mode, which aims to prevent the error propagation. The image is a two-dimensional subband decomposed using Daubechies 16 wavelet filters, while every two consecutive video frames are 3-D subband decomposed [15], [16] together using Harr filtering in time domain and wavelet filtering in spatial domain. Targeted average bit rates range from 0.3 to $1.0 \mathrm{bpp}$. For the Salesman image and video sequences with a frame size of $360 \times 288$, these correspond to 31 104-103680 bits/frame. The peak signal-to-noise ratio (PSNR) is used to measure the quality of decoded frames.

The source quantization for the lowest subband, which represents the dc component, is different from the other subbands. We use two sets of methods in the simulation:

- Method A: uniform quantizer on lowest subband and tree search vector quantization of four dimensions on other subbands;

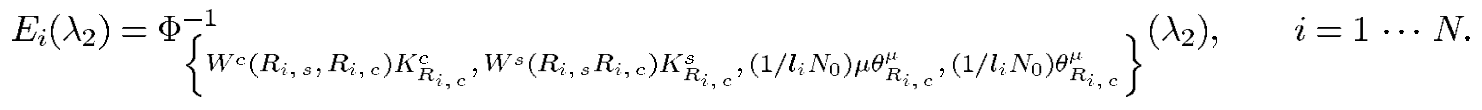




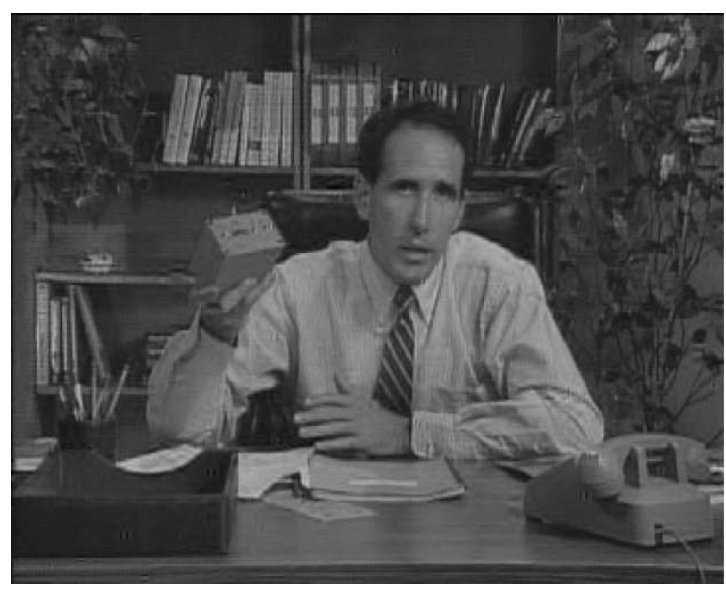

(a)

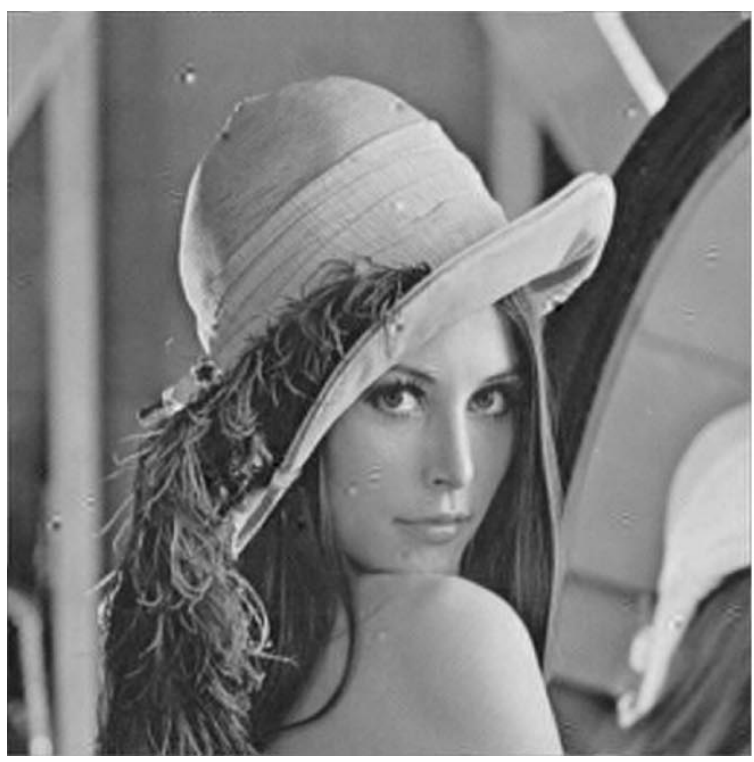

(c)

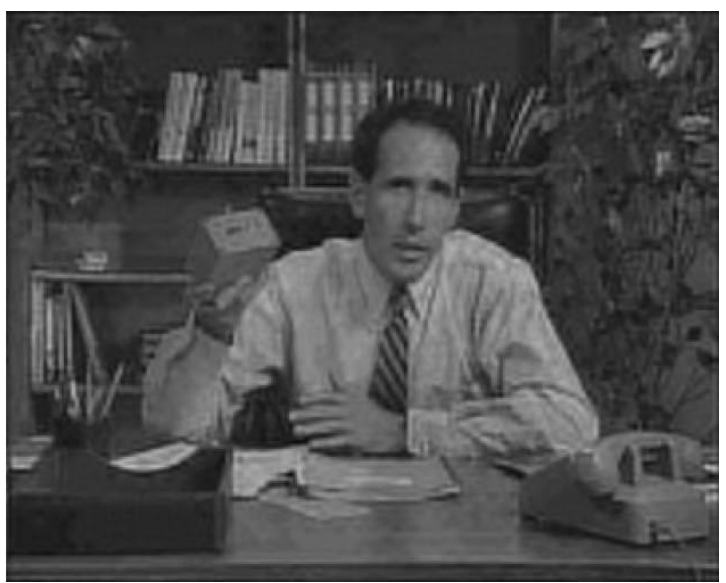

(b)

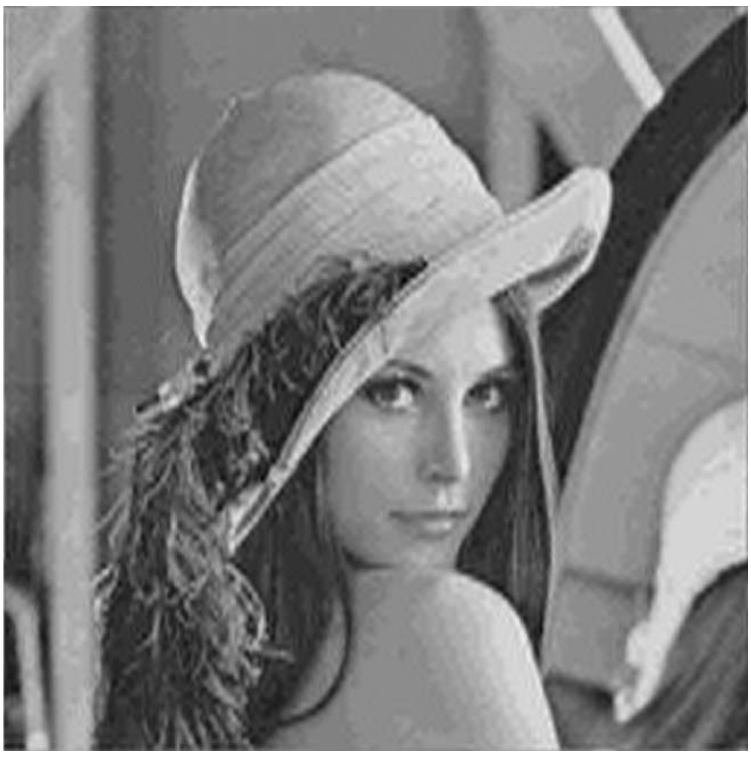

(d)

Fig. 14. Comparison between (a) and (c) proposed system and (b) and (d) fixed BPSK modulation system at SNR $=15$ dB.

- Method B: Lloyd quantizer on lowest subband and full search VQ of four dimensions on the other subbands.

The available modulations are BPSK, MR-PAM-4, MR-QAM16, and MR-QAM-64, as illustrated in Fig. 2. We simulate the system by using the distortion computation described in Section IV-C, referred to as the complex approach, and by using distortion approximation in Section $\mathrm{V}$, the approximation approach. In the complex approach, transmitted power is distributed equally among the subbands to be transmitted. For comparison, we derive the results for both equal power and power allocation using approximation approach.

We use one frame from the Salesman sequence as an image example. The PSNR versus channel SNR performance is shown in Fig. 8 using the complex approach. When the channel SNR is less than $5 \mathrm{~dB}$, the performance difference for different source rates is small. Since the noise effect is large, only BPSK is employed. Increasing source rate will decrease the source distortion. However, it may increase the channel distortion at the same time. As a result, the overall performance will not improve much. In the middle SNR range, which is of our interest, there is a higher PSNR difference, given the different source rate, although here the video is becoming perceptually objectionally corrupted.

Fig. 9 compares the complex and approximation approach. As can be seen, distortion approximation with equal power distribution performs nearly as the same as the complex approach. This further proves that the channel distortion is well approximated. Power allocation allows the error performance to be assigned more efficiently to the subbands according to their perceptual importance. It then achieves $0.4-2 \mathrm{~dB}$ performance improvement. The improvement increases as the source rate increases.

Fig. 10(a) shows the impact of factor $\mu$ on the performance. As in distortion analysis (25), $\mu$ controls the error performance of the clouds and satellites, and therefore the error performance of MSB's and LSB's of source codewords. Note that $d=\theta_{R_{i, c}}^{\mu} \sqrt{E}$ and $\theta_{R_{i, c}}^{\mu}$ is a monotonic function of $\mu$. Increasing $\mu$ results in smaller $d$ for fixed $E$, and therefore more difference between the error performance of clouds and satellites. The optimal $\mu$ depends on $W^{s}($.$) and W^{c}($.$) . For$ 
fixed transmitted power, larger $W^{c}(.) / W^{s}($.$) requires larger$ $\mu$. Or equivalently, the selection of $\mu$ is source oriented. Therefore, different subbands are associated with different $\mu$. However, this introduces extensive computations as well as an increased complexity at the transmitter/receiver. For simplicity, we choose to fix $\mu$ throughout the transmission of a single image or video sequence. As shown in Fig. 10, $\mu=1.2$ achieves better performance than $\mu=1.0$ and $\mu=1.5$.

We plot the PSNR performances of the proposed system using direct mapping, bit remapping, and the optimal mapping, shown in Fig. 10(b). Direct mapping takes the bits consecutively and maps them to the constellation points. The optimal mapping is obtained as the optimal solution of (7). As can be seen, the bit-remapping system performs nearly the same as the optimal mapping system and is about $0.2-1.6 \mathrm{~dB}$ better than the system without mapping design, particularly in the middle SNR range.

To demonstrate the advantage of combined source coding and channel modulation design over the separate designs, we compare the proposed system to the fixed modulation systems. In the fixed modulation systems, the subbands are transmitted using the same modulation, and the rate allocation is applied to the source rate. Therefore, the source coding and channel modulation are designed separately. We do not use power allocation to make clear the advantage of joint design over separate design. The corresponding image and video results are shown in Figs. 11 and 12. As can be seen, in general, when the channel SNR is less than $8 \mathrm{~dB}$, the proposed system has the same performance as that of the BPSK modulation system, since the only suitable modulation for this low SNR range is BPSK. As the channel SNR increases, the channel error is negligible for some modulations. For example, when the channel SNR equals $10 \mathrm{~dB}$, the BER of BPSK is fairly small. Selecting BPSK to transmit all the subbands results in large source distortion, although the channel distortion is small. On the other hand, transmitting all the subbands using PAM-4 will induce large channel distortion. In such a case, assigning BPSK to some important subbands and PAM-4 or modulation with higher rate to others may achieve better performance. Such assignment aims to compensate the increase in the channel distortion by reducing the source distortion. We employ MR modulation and bit remapping in both the fixed modulation systems and the proposed system. Examining the visual quality of the proposed system and the BPSK system from Fig. 14, the images generated by the proposed system are clearer and more detailed, with only a negligible number of defective points. It can be concluded that the proposed combined source coding and modulation system performs substantially better than fixed modulation systems, especially at low transmission bandwidth scenarios.

We also compared the proposed system to the other two existing approaches [3], [4]. Fig. 13(a) compares the proposed system with the one-to-one mapping system of [4], in terms of PSNR versus SNR curve for the $512 \times 512$ Lena image coded at 0.5 and $0.4 \mathrm{bpp}$. For channel SNR's ranging from 10 to $24 \mathrm{~dB}$, the proposed system shows a better performance. When the channel SNR is above $24 \mathrm{~dB}$, the proposed system saturates due to the upper bound on source coding rate. For the comparison in very low SNR scenarios, such as 1-8 $\mathrm{dB}$, we also plot the performance of the joint source and channel coding system with RCPC code on the Salesman video sequence [3]. Fig. 13(b) illustrates that for very low SNR (below $6 \mathrm{~dB}$ ), using channel coding with BPSK generates preferable results. When the channel SNR is above $6 \mathrm{~dB}$, the proposed system shows great improvement by assigning modulation at different rates to different subbands. It also shows that the proposed system can achieve nearly the same performance at much lower rates for some higher SNR values. The above two comparisons further prove that the proposed system is most suitable for channels exhibiting medium (8-22 dB) SNR values and low bandwidths.

\section{CONCLUSION}

We have proposed a combined source coding and channel modulation scheme for image and video transmission over noisy channels that is simple, efficient, and robust against channel errors. The flexibility of combined source coding and channel modulation design as well as various compression capabilities and UEP of multilevel MR modulation were fully utilized. The proposed bit-remapping scheme allows the MSB's of a source codeword to be mapped to the cloud bits of MR modulation as much as possible; thus the MSB's receive better protection than the LSB's. We derive a simple optimization function that can employ power allocation to further improve the performance. The system performed significantly better than other schemes proposed in the literature on the AWGN channel exhibiting moderate SNR and low bandwidth. Compared to conventional schemes, the proposed scheme needs three or four modulators/demodulators. Since we use common modulations such as BPSK, PAM, and QAM, which are quite the same in terms of implementation, the increase in complexity is negligible compared to the performance improvement.

\section{REFERENCES}

[1] Y. Shoham and A. Gersho, "Efficient bit allocation for an arbitrary set of quantizers," IEEE Trans. Acoust., Speech, Signal Processing, vol. 36, pp. 1443, 1453, Sept. 1988.

[2] G. Cheung and A. Zakhor, "Joint source/channel coding of scalable video over noisy channel," in Proc. Int. Conf. Image Processing, 1996, pp. 767-770.

[3] M. Srinivasan, P. Burlina, and R. Chellappa, "Adaptive source-channel subband video coding for wireless channels," in Proc. 1st IEEE Workshop Multimedia Signal Processing, Princeton, NJ, June 1997.

[4] J. M. Lervik and T. A. Ramstad, "Robust image communication using subband coding and multilevel modulation," in Proc. SPIE VCIP'96, 1996, vol. 2727 , pp. 524-535.

[5] J. Hagenauer, "Rate-compatible punctured convolutional codes (RCPC codes) and their applications," IEEE Trans. Commun., vol. 36, pp. 389-399, Apr. 1988.

[6] I. Kozintsev and K. Ramchandran, "Multiresolution joint source-channel coding using embedded constellations for power-constrained timevarying channels," in Proc. Int. Conf. Image Processing, 1996, pp. 2343-2346.

[7] J. W. Woods and S. D. O'Neil, "Subband coding of images," IEEE Trans. Acoust., Speech, Signal Processing, vol. 34, pp. 1278-1288, Oct. 1986.

[8] N. Farvadin and V. Vaishampayan, "Optimal quantizer design for noisy channels: An approach to combined source and channel coding," IEEE Trans. Inform. Theory, vol. 22, pp. 461-470, 1986.

[9] J. G. Proakis, Digital Communications, 3rd ed. New York: McGrawHill, 1995. 
[10] G. Ungerboeck, "Channel coding with multi-level/phase signals," IEEE Trans. Inform. Theory, vol. 18, pp. 55-66, 1982.

[11] K. Ramchandran and M. Vetterli, "Best wavelet packet bases in a ratedistortion sense," IEEE Trans. Image Processing, vol. 2, pp. 160-174, Apr. 1993.

[12] W. T. Webb and L. Hanzo, Modern Quadrature Amplitude Modulation, Principles and Applications for Fixed and Wireless Channels. New York: Pentech and IEEE Press, 1994.

[13] L. F. Wei, "Coded modulation with unequal error protection," IEEE Trans. Commun., vol. 41, pp. 1439-1449, Oct. 1993.

[14] A. R. Calderbank and N. Seshddri, "Multilevel codes for unequal error protection," IEEE Trans. Inform. Theory, vol. 39, pp. 1234-1248, July 1993.

[15] D. Taubman and A. Zakhor, "Multirate 3-D subband coding of video," IEEE Trans. Image Processing, vol. 3, pp. 572-588, Sept. 1994.

[16] C. I. Podilchuk, N. S. Jayant, and N. Farvardin, "Three-dimensional subband coding of video," IEEE Trans. Image Processing, vol. 4, pp. 125-139, Feb. 1995.

[17] H. Zheng and K. J. R. Liu, "Image and video transmission over wireless channel: A subband modulation approach," in IEEE Proc. Int. Conf. Image Processing, Chicago, IL, Oct. 1998.

[18] "Robust image and video transmission over spectrally shaped channels using multicarrier modulation," IEEE Trans. Multimedia, vol. 1, no. 1, pp. 88-103, 1999.

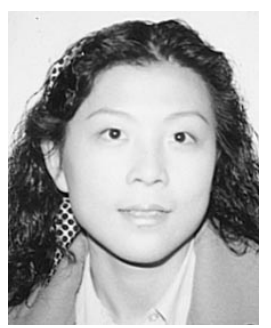

Haitao Zheng received the B.S. degree from Xian Jiatong University, Xian, China, in 1995 and the M.S. degree from the University of Maryland, College Park, in 1998, both in electrical engineering. She is currently pursuing the Ph.D. degree at the University of Maryland.

From 1994 to 1995, she was a Member of the Video Technology Institute, Xian Jiaotong University. From 1995 to 1998 , she was an Institute for System Research Fellow at the University of Maryland, College Park. Her research interests include multimedia communications, multicarrier modulation, and wireless communications.

Ms. Zheng received the 1998-1999 George Harhalakis Outstanding Systems Engineering Graduate Student Award from the University of Maryland in recognition of her outstanding contributions in cross-disciplinary research.

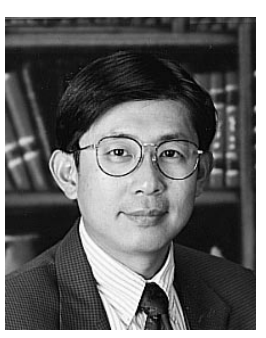

K. J. Ray Liu (S'86-M'90-SM'93) received the B.S. degree from the National Taiwan University, Taiwan, R.O.C., in 1983 and the Ph.D. degree from the University of California, Los Angeles, in 1990, both in electrical engineering.

Since 1990, he has been with the Electrical Engineering Department and Institute for Systems Research, University of Maryland at College Park, where he is an Associate Professor. During his sabbatical leave in 1996-1997, he was a Visiting Associate Professor at Stanford University, Stanford, CA. His research interests span various aspects of signal/image processing and communications. He has published more than 160 papers, of which 60 are in archival journals and book chapters. He is an Editor of the Journal of VLSI Signal Processing Systems and the Book Series Editor of a Marcel Dekker series on signal processing. He is a co-editor of High Performance VLSI Signal Processing, Volume I: System Design and Methodology and Volume II: Algorithms, Architectures, and Applications (New York: IEEE Press, 1998).

Dr. Liu has received numerous awards, including the 1994 National Science Foundation Young Investigator Award; the IEEE Signal Processing Society's 1993 Senior Award (Best Paper); and the George Corcoran Award in 1994 for outstanding contributions to electrical engineering education and the 1995-1996 Outstanding Systems Engineering Faculty Award in recognition of outstanding contributions in interdisciplinary research, both from the University of Maryland. He has been an Associate Editor of IEEE Transactions on Signal Processing. He was a Guest Editor of special issues on "Multimedia Signal Processing and Technology" of the ProceEDINGS OF THE IEEE, a Guest Editor of a special issue on "Signal Processing for Wireless Communications" of the IEEE Journal on Selected AREAS IN Communications, and the Chair of the Multimedia Signal Processing Technical Committee of the IEEE Signal Processing Society. 